\title{
Designing bending-active gridshells as falsework for concrete shells through numerical optimization
}

\author{
Jef Rombouts ${ }^{a, b}$, Andrew Liew ${ }^{c}$, Geert Lombaert ${ }^{d}$, Lars De Laet ${ }^{b}$, Philippe Block ${ }^{c}$, Mattias Schevenels ${ }^{a}$ \\ ${ }^{a}$ KU Leuven, Faculty of Engineering Science, Department of Architecture, Kasteelpark Arenberg 1 - box 2431, 3001 Leuven, Belgium \\ ${ }^{b}$ Vrije Universiteit Brussel, Faculty of Engineering, Department of Architectural Engineering \\ ${ }^{c}$ ETH Zurich, Institute of Technology in Architecture, BLOCK Research Group \\ ${ }^{d}$ KU Leuven, Faculty of Engineering Science, Department of Civil Engineering
}

\begin{abstract}
Shell structures are material efficient structures capable of covering large spans with minimum weight. If concrete is used as the building material for the shell, it must be initially supported by a formwork. These generally rigid formworks, and their supporting falsework structure, are time and material consuming to construct. Recently, researchers have recommended the use of elastic gridshells as falsework system. Unfortunately, the design of such a system is complicated by large deformations of the bending elements. Moreover, since gridshells are inherently flexible, significant displacements generally occur when applying the wet concrete.

To overcome these difficulties, this paper presents a design tool to effectively design a gridshell serving as the falsework for a concrete shell. The gridshell is assumed to be constructed from an initially flat grid of straight slender rods, using cables with adjustable lengths to brace the erected gridshell. An optimization algorithm is proposed that fits the shape of the gridshell under the wet concrete loading to a given target shape by manipulating the lengths of the bracing cables. Meanwhile, the optimization algorithm reduces the construction effort by minimizing the number of cables, and the diameter of the grid rods. Moreover, feasibility of the design is ensured by constraining the axial force in the cables and limiting the displacements under increased loading. A gradient-based optimization scheme is adopted, and an implicit dynamic relaxation approach previously developed specifically for the simulation of bending-active structures is used to solve the nonlinear equilibrium equations. Nonlinear effects such as buckling of the grid rods are taken into account by using co-rotational beam elements. The resulting design tool is applied for the design of a scale model, demonstrating the feasibility of the suggested falsework system. The optimized design fits the target better, uses less cables, and is more resistant to additional loading compared to designs from existing design approaches. Comparisons between the numerical and physical models show deviations of up to around $2 \%$ of the larger span for the design load and smaller deviations for smaller loads, with differences attributed to small model scaling effects.
\end{abstract}

Keywords: bending-active gridshells, active bending, numerical optimization, flexible formwork, concrete shells

\section{Introduction}

Shell structures are material efficient structures capable of covering large spans with minimum weight. When they are properly designed and built, internal bending moments are minimized or avoided altogether, and the external loads are carried exclusively through membrane action. The extent to which the structure acts as a membrane rather than a plate is determined by the shape of the shell. This shape needs some curvature, preferably in all directions to attain stiffness through its geometry. This makes on-site construction more challenging. If concrete is used as the building material for the shell, a full and rigid formwork is usually required to hold the wet concrete before the structure can carry its own weight. These rigid formworks, and their supporting falsework structure, are time and material consuming to make, as most of the formwork components have to be customized to fit the required shape. Consequently, despite their inherent material efficiency, concrete shells are usually too expensive to build in practice.
With the aim of making concrete shells a more viable structural solution, researchers have been working on the development of flexible formwork instead of traditional rigid formwork to reduce the time and material spent on the construction of the shell. Flexible formwork generally consists of a membrane on which the concrete is cast. If the membrane is not strong enough to span the whole surface by itself, falsework or air pressure is required to support and shape the membrane. The falsework consists of rigid elements, cables, bending-active elements, or a combination of these. Because this formwork is inherently flexible, a common challenge is to limit the deformations of the formwork under the weight of the wet concrete. This challenge is usually addressed by applying the concrete in layers, gradually increasing stiffness and weight. Moreover, because small variations in shape can cause a large drop in performance of the concrete shell $[1,2]$, it is important for the formwork to accurately match the designed shape. A short overview of each type of flexible formwork is given below. 
1. Membrane formwork supported by rigid elements already leads to a high reduction in construction effort and material use compared to rigid formwork. As a result, this type of formwork quickly became very popular when it was first applied during the second world war by Waller and Billig [3]. Their Ctesiphon system uses jute fabric stretched over a series of rigid arches. However, the rigid elements can only be reused in near identical designs, which limits the adaptability and design freedom of this system.

2. Pneumatic formwork has the advantage that it is light, compact, re-usable and fast to erect. A comprehensive overview of the history of pneumatic formwork is given by Kromoser and Huber [4]. The main challenge related to this type of formwork is the large deformation caused by the weight of the wet concrete [5], which is detrimental for the structural performance of the final structure [6]. Moreover, Hennik and Houtman [7] showed that designing a pneumatic formwork for a given target shape is far from trivial. A final disadvantage is that pneumatic formwork covers the whole construction area underneath the shell during casting and curing of the concrete, which makes this system unusable for use over existing structures, rivers, or roads that have to remain operational during construction.

3. Membranes supported by cable nets do not obstruct the space underneath the shell during construction. Van Mele and Block [8], and Veenendaal and Block [9] developed a design approach for this type of formwork. Motivated by the fact that the structural properties of a shell are potentially very sensitive to their shape, they employed numerical optimization to fit a cable-net to a target shape, while taking the weight of the wet concrete into account. Later, Liew et al. [10] extended the approach to adaptively account for design imperfections. With a large prototype for an actual roof, Block et al. [11, 12] showed the potential of this type of formwork. However, a drawback is its limitation to anticlastic shapes, while shells acting in pure compression under gravitational loading generally have some form of synclastic curvature.

4. The use of bending-active elements in formwork for concrete shells is relatively new. Popescu et al. [13] incorporated just a few bending-active elements in a knitted flexible formwork. Tang and Pedreschi [14], Coar et al. [15] and Cuviliers et al. [16] on the other hand, proposed the use of bending-active gridshells as (lost) falsework. Because bending-active gridshells are generally erected from an initially flat grid of continuous rods, with pinned connections between crossing rods, they do not require the production of specially customized beams or joints. This simplifies the construction process and increases the reusability of the components. Moreover, the flexibility of the rods, in combination with the shear degree of freedom provided by the pinned connections, allows the structure to be deformed into complex curved shapes which can have both anticlastic and synclastic curvature. Consequently, these structures have high potential to be used as falsework for concrete shells, and they also allow to keep the construction area underneath the shell free. However, despite their construction efficiency, their design is complicated by the large deformation associated with the bending erection process.

Hernández [17] compared several design approaches to fit a gridshell to a given target shape. However, because a gridshell is usually allowed to relax its shape before an additional bracing layer 'fixes' that shape by eliminating the initial shear degree of freedom, significant deviations from the intended target shape can be expected. Moreover, if the gridshell is to be used as the falsework for a concrete shell, the weight of the wet concrete will most likely cause even larger deformations and deviations from the target shape. The deviations due to relaxation can be counteracted by using the bracing to fix the shape of the gridshell before it is allowed to relax. Alternatively, Pignatelli et al. [18] proposed a genetic algorithm to find the shaping forces needed to push or pull the grid towards the intended target after the grid has been erected. However, to our best knowledge, design tools taking deformations due to loading into account are still lacking.

This paper presents a framework for the design of bendingactive gridshells as a falsework for concrete shells. As a key part of the design framework, a novel optimization method is developed to fit the supporting gridshell to a given target shape by accurately simulating the bending process, while taking the wet concrete loading into account to make sure the gridshell fits the exact target shape in its loaded condition. The cross-section of the grid rods and the undeformed lengths of the braces are chosen as the design variables. The design framework assumes full circular cross-sections for the grid rods, and a bracing system with adjustable lengths, using for example turnbuckles or lashing straps, to manipulate the gridshell's shape after its relaxation. In contrast to the approach proposed by Pignatelli et al. [18], our approach requires no additional external struts or ties to shape the grid, as we use the bracing not only to stiffen the gridshell, but also to manipulate its shape after erection. This enables us to keep the space underneath the shell free of structural elements. Additionally, the number of braces and the radius of the cross-section of the grid rods are minimized, and feasibility of the design is ensured by constraining the axial force in the braces. Finally, the robustness of the optimized design with respect to load uncertainties is improved by limiting the displacements under an additional load case. The proposed design approach is tested by designing and building a structurally representative scale model.

In a recently published paper, the authors describe a novel shape optimization approach for bending-active gridshells [19]. The objective of the current paper is different, but the setup of the structural model is identical. Hence, also small parts of the sensitivity analysis are identical. Nevertheless, both the structural model and the sensitivity analysis are described in full in the current paper (the latter in the appendix) for a better comprehensibility.

The remainder of this paper is organized as follows. First, section 2 gives an overview of all steps in the proposed design framework for a gridshell acting as the falsework for a concrete shell. Next, section 3 explains how the gridshell is modeled using finite elements. Section 4 discusses the design and construction of a scale model, and finally, conclusions are given in section 5 . 


\section{Design framework}

This section describes the framework for the design of bending-active gridshells as the falsework for concrete shells. The goal of this framework is to design a gridshell that closely approximates a given target shape under the weight of the wet concrete. Additionally, the construction effort and material use should be minimized by reducing the number of braces and the cross-section of the grid rods. Moreover, the bending moments in the rods and normal forces in the braces should be limited to ensure that the design is feasible. Finally, the design should be robust by limiting deviations from the target shape under increased loading. The design framework assumes a regular grid, round rod sections, and a bracing system with adjustable lengths. Moreover, the bracing elements are assumed to only withstand tensile forces. Consequently, they are simply referred to as cables in the following.

A flowchart of the proposed design framework is given in figure 1. The design process consists of two parts. In the first part, the initial design is generated, following a similar approach to the one proposed by Pignatelli et al. [18]: a so-called Chebyshev net is drawn on the target surface to extract the dimensions and layout of the corresponding flat grid, locations of the supports and maximum cross-section of the grid rods. This information is used to generate the initial design. Moreover, the Chebyshev net is used as a target grid in the second part, where the undeformed length of the cables and the cross-section of the grid rods are optimized so that the resulting gridshell fits the target shape under the wet concrete loading. Each step in the flowchart is explained in the following paragraphs.

\section{Input parameters}

The target shape is determined in advance by the designer as the desired shape for the actual concrete shell. This can be any smooth shape, with both positive and/or negative Gaussian curvature, as long as the maximum curvature does not exceed the maximum achievable curvature of the used rods. However, since the rods' cross-section is determined later in the design framework, it is not possible to know the maximum allowable curvature in the shell beforehand. Additionally, the designer must choose the distance between grid rods, based on practical considerations. Next, the mechanical properties of the cables should be determined. Finally, the designer must choose a catalogue of available rod sections.

\section{(1) Generation of Chebyshev net}

The first operation of the design framework is to draw a potential grid or Chebyshev net on the target surface. Several techniques are described in the literature, which can be categorized into two groups [17]. Methods in the first group treat the problem as a purely geometrical problem. The most commonly used method in this group is the compass method, originally proposed by Frei Otto [20], which requires the designer to choose a mesh density, and two intersecting curves on the surface. Other methods use numerical optimization to draw a grid on [21, 22], or near the target surface [23], allowing additional control over some grid characteristics such as the curvature of the

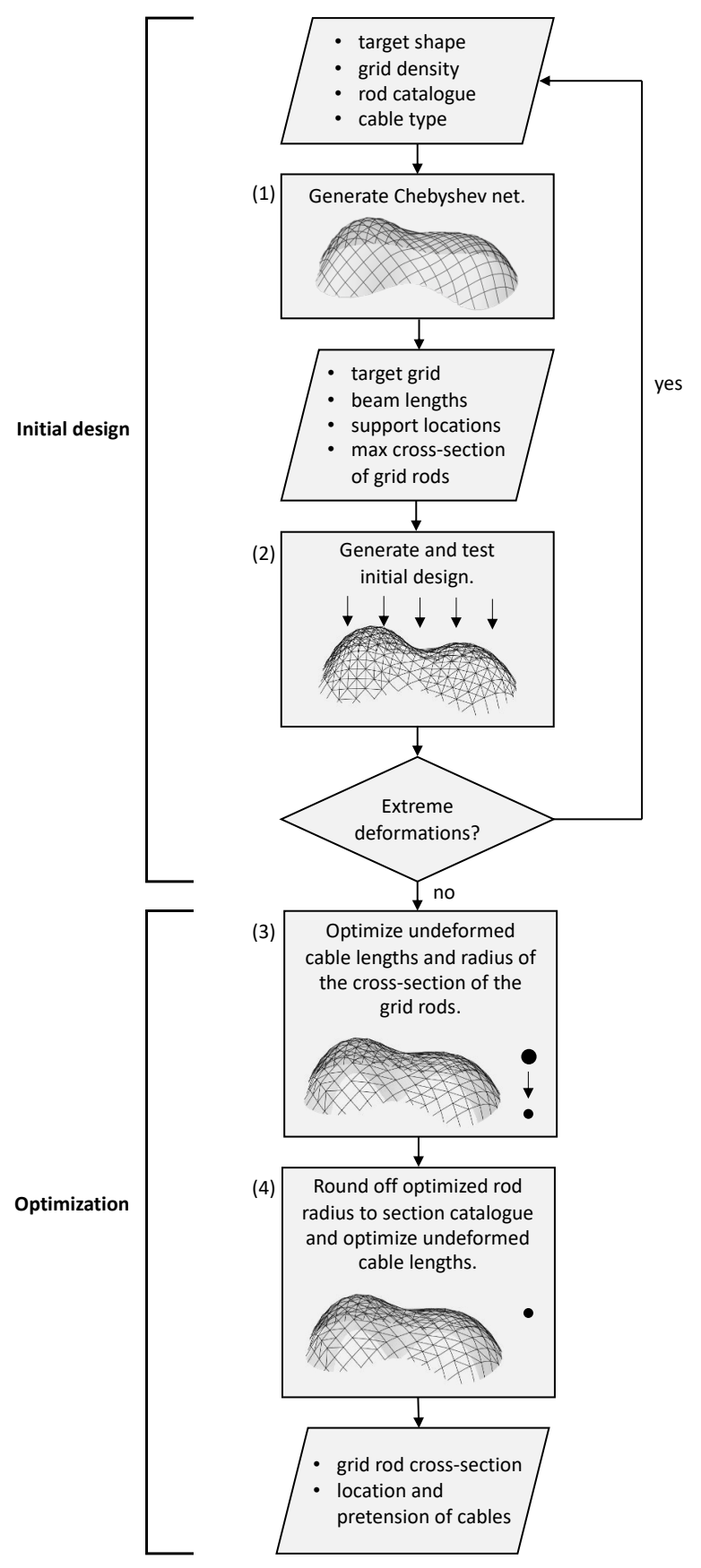

Figure 1: Flowchart of the proposed design framework. 
lines or angle between line segments. The second group treats the problem as a fictitious physical problem, pulling or pushing a flat grid towards the target surface using fictitious forces [24], springs [25], or geometric constraints [26]. In this paper, we follow Kuijenhoven and Hoogenboom [25] as we pull an over-sized grid towards the target surface with fictitious springs, trimming excess parts, and using implicit dynamic relaxation to solve the nonlinear equilibrium equations [27]. Compared to a purely geometrical approach, this approach has the advantage that the grid has a certain stiffness. Consequently, the grid will relax to a state of low deformation energy, as the rods try to straighten out as much as possible on the surface. Additionally, the stiffness of the connections can be tuned to control the angle between crossing rods. Furthermore, the forces in the cables can be used to determine to what extent the grid is forced to the target surface. However, the fact that the material properties are entirely fictitious at this stage makes it hard to directly choose appropriate values for these properties. A trial and error approach is often required to obtain the desired geometry. For this reason, other techniques are potentially worth considering at this stage.

The resulting Chebyshev net is used to determine the length and connectivity of each rod in the grid, the locations of the supports, and the maximum allowable cross-section, derived from the maximum curvature in the grid. The maximum allowable cross-section can be derived directly from the internal forces generated by the nonlinear finite element solver. Assuming a circular cross-section, the maximum allowable radius for the grid rods is given by:

$$
r_{\mathrm{g}, \max }=\frac{I f_{\mathrm{f}}}{\sqrt{M_{y}^{2}+M_{z}^{2}}}
$$

where $I$ is the moment of inertia, $f_{\mathrm{f}}$ is the longitudinal flexural strength, and $M_{y}$ and $M_{z}$ are the bending moments around both principal axes. Additionally, the Chebyshev net will serve as the target grid in the second part of the design framework.

\section{(2) Initial design}

The second operation entails the generation of an initial design, using the grid layout and support locations from the previous step, and assigning the maximum allowable cross-section to all grid rods. Moreover, cables are modeled at all allowable positions. The cables are given a certain level of prestress by setting their undeformed lengths $\mathbf{l}_{\mathrm{c}}^{0}$ to a fraction of their current lengths $\mathbf{l}_{\mathrm{c}}\left(\right.$ e.g. $\mathbf{l}_{\mathrm{c}}^{0}=0.99 \mathbf{l}_{\mathrm{c}}$ ), which is defined as the distances between the nodes they connect in the target grid, generated in the previous step. This layout of cables serves as a ground structure (i.e. a structure containing all possible cable elements) for the optimization in steps 3 and 4 . In order for the optimization to converge, the loaded gridshell in its current, initial design should be close enough to the target grid: it should not show excessive deformations like global snap-trough buckling under the wet concrete loading. If excessive deformations do occur, a denser grid should be chosen in the first step.

\section{(3) Optimization of rod cross-section and cable lengths}

If the deformations of the initial design under loading are acceptable, the prestress of the cables and cross-section of the rods can be optimized. This third operation in the flowchart forms the key aspect of the design framework. The optimization problem is formulated as follows:

$$
\begin{array}{ll}
\min _{\mathbf{l}_{\mathrm{c}}^{0}, r_{\mathrm{g}}} & f=w_{1} \mathbf{d}^{\mathrm{T}} \mathbf{d}+w_{2} \sum_{i=1}^{k_{\mathrm{c}}} n_{\mathrm{c}, i}+w_{3} r_{\mathrm{g}} \\
\text { s.t. } & \mathbf{l}_{\mathrm{c}, \min }^{0} \leq \mathbf{l}_{\mathrm{c}}^{0} \leq \mathbf{l}_{\mathrm{c}, \max }^{0} \\
& r_{\mathrm{g}, \min } \leq r_{\mathrm{g}} \leq r_{\mathrm{g}, \max } \\
& \mathbf{n}_{\mathrm{c}} \leq \mathbf{n}_{\mathrm{c}, \max } \\
& \mathbf{d}_{1}^{\mathrm{T}} \mathbf{d}_{1} \leq d^{2}
\end{array}
$$

where the undeformed length of each cable, collected in a vector $\mathbf{l}_{\mathrm{c}}^{0}$ and the radius of the cross-section of all grid rods $r_{\mathrm{g}}$ are the design variables, $w_{1}, w_{2}$, and $w_{3}$ are weighting factors, $\mathbf{d}$ is a vector collecting the distances between each node and the corresponding node in the target grid, $n_{\mathrm{c}, i}$ is the normal force in cable $i$ and the $i$-th element of $\mathbf{n}_{\mathrm{c}}, k_{\mathrm{c}}$ is the number of cables, $\mathbf{l}_{\mathrm{c}, \min }^{0}$ and $\mathbf{l}_{\mathrm{c}, \text { max }}^{0}$ are vectors collecting the minimal and maximal initial length for each cable, $r_{\mathrm{g}, \min }$ and $r_{\mathrm{g}, \max }$ are the minimal and maximal cross-section radius, given by the chosen section catalogue, $\mathbf{n}_{\mathrm{c}, \max }$ is a vector collecting the maximum allowable tensile force in each cable, $\mathbf{d}_{1}$ is a vector collecting the distances between each node and its corresponding node in the target grid for an additional load case, and $d^{2}$ is a maximum allowable value for $\mathbf{d}_{1}^{\mathrm{T}} \mathbf{d}_{1}$. The distances collected in vectors $\mathbf{d}$ and $\mathbf{d}_{1}$ are measured as the Euclidian distances between the current grid nodes and the nodes of the target grid generated in the first step of the design framework. For node $i$ :

$$
d_{i}=\sqrt{\left(x_{i}-x_{i}^{\mathrm{t}}\right)^{2}+\left(y_{i}-y_{i}^{\mathrm{t}}\right)^{2}+\left(z_{i}-z_{i}^{\mathrm{t}}\right)^{2}}
$$

where $d_{i}$ is the distance of node $i$ and the $i$-th element of vector d, $x_{i}, y_{i}$, and $z_{i}$ are the $\mathrm{x}-, \mathrm{y}^{-}$, and $\mathrm{z}$-coordinates of node $i$, and $x_{i}^{\mathrm{t}}$, $y_{i}^{\mathrm{t}}$, and $z_{i}^{\mathrm{t}}$ are the $\mathrm{x}-, \mathrm{y}-$, and $\mathrm{z}$-coordinates of the corresponding target node.

Equation (2) shows that the objective is threefold; first, the goal is to minimize the distance between the loaded gridshell and the target grid. Second, the number of cables is penalized using $\mathrm{L} 1$ regularization, which effectively reduces the tension in unnecessary cables to zero. Third, the rod section is minimized to reduce the material use and the construction effort by reducing the magnitude of the erection forces required to bend the grid into its final shape. The share of each part of the objective function is managed by tuning the weighting factors $w_{1}$, $w_{2}$, and $w_{3}$, for which the following units are chosen: $w_{1}\left[\mathrm{~m}^{-2}\right]$, $w_{2}\left[\mathrm{~N}^{-1}\right], w_{3}\left[\mathrm{~m}^{-1}\right]$. This results in a dimensionless objective function.

Box constraints on the undeformed cable lengths $\mathbf{l}_{\mathrm{c}}^{0}$ (equation (3)) can be included to improve convergence by limiting the design domain. Box constraints on the radius $r_{\mathrm{g}}$ of the crosssection of the rods (equation (4)) ensure that the optimized rod section is within the bounds of available cross-sections of the 
chosen section catalogue. A constraint on the normal force in the cables $\mathbf{n}_{\mathrm{c}}$ (equation (5)) ensures that the design is feasible. Finally, a constraint on the deviation from the target grid under an additional load case (equation (6)) makes the design more robust with respect to load variations. If needed, similar constraints for additional load cases can be added to the optimization problem, e.g. to account for non-uniform loading during the pouring of concrete if it is not applied in layers. After the optimization process converges, cables that are not tensioned under the design load are removed from the numerical model.

The optimization problem is solved by means of a gradientbased optimizer. The gradients of the terms in the objective function and the constraints are determined analytically. This sensitivity analysis is described in appendix A, along with a verification of the resulting expressions by means of the finite difference method.

\section{(4) Optimization of cable lengths}

Although the radius of the cross-section of the rods is a discrete variable in reality, it is optimized as a continuous variable. Consequently, the optimized radius must be rounded to the nearest higher radius available in the chosen section catalogue. This may affect the performance and feasibility of the optimized gridshell. Therefore, as a fourth and final operation in the design framework, the initial lengths of the cables are optimized again with the same objective and constraints, while keeping the cross-section of the rods fixed. Rounding the sections up makes the grid stiffer, which means more cables and/or larger cable forces are needed to deform the grid towards the target shape. On the other hand, the grid will be less sensitive to additional load cases. Therefore, it is likely that a feasible solution can be found in this step if a feasible solution was found in the previous step, while the objective function increases slightly. As an extension to the proposed method, one might consider the use of a penalization or projection strategy to steer the continuous rod section towards a discrete solution - this might lead to a better optimum than rounding and reoptimizing. However, as opposed to penalization or projection strategies, rounding and re-optimizing ensures that a perfectly discrete solution is obtained.

\section{Finite element model}

The behavior of the gridshell is modeled with nonlinear finite elements, using implicit dynamic relaxation to iteratively find the structure's equilibrium position. For more details on implicit dynamic relaxation, which has been developed specifically for the simulation of bending-active structures, the reader is referred to [27] and [28].

The structure is in equilibrium when the external loads are balanced by the internal forces, i.e. all residual forces are zero:

$$
\mathbf{r}=\mathbf{p}-\mathbf{f}=\mathbf{0}
$$

where $\mathbf{r}$ are the residual forces, $\mathbf{f}$ are the internal forces, and $\mathbf{p}$ are the external loads. In practice, the residual forces will never be exactly zero, and the structure is assumed to be in equilibrium when the solver reduces the residual forces below a certain threshold. The grid rods are modeled using co-rotational beam elements [29], a short description of which is given next in section 3.1. In order to account for the eccentricity of crossing rods, two separate nodes are defined for every connection. The connections are modeled as short co-rotational beam elements between these nodes, with low or no torsional stiffness to allow rotations in the plane of the grid during erection. These elements do have non-zero axial and bending stiffness - the actual values must be determined experimentally. Cables are modeled as beam elements with no torsional or bending stiffness, and no stiffness in compression.

\subsection{Co-rotational beam elements}

Co-rotational beam elements are specifically developed to handle large displacements [30]. They allow arbitrarily large translations and rotations, as long as the strains remain small. This is achieved by decoupling rigid body motion and local beam deformations. The basis of the co-rotational beam element as described by Crisfield [29] is briefly reviewed here. A more extensive explanation can be found in [29] or [31].

To distinguish between rigid body motion and local beam deformations, a local co-rotated reference configuration is fitted to the actual deformed beam. Local deformations are traced by comparing the actual deformed beam to the co-rotated reference beam. The co-rotated reference beam is represented by a set of local element basis vectors $\mathbf{T}_{\mathrm{e}}=\left[\mathbf{e}_{x} \mathbf{e}_{y} \mathbf{e}_{z}\right]$, where $\mathbf{e}_{x}$ lies in the direction of the vector connecting the beam ends. This set of element basis vectors is constructed from the known current beam end orientations, which are in turn represented by the nodal basis vectors $\mathbf{T}_{\mathrm{a}}=\left[\begin{array}{lll}\mathbf{a}_{x} & \mathbf{a}_{y} & \mathbf{a}_{z}\end{array}\right]$ and $\mathbf{T}_{\mathrm{b}}=\left[\begin{array}{lll}\mathbf{b}_{x} & \mathbf{b}_{y} & \mathbf{b}_{z}\end{array}\right]$ (figure 2). Note that $\mathbf{a}_{x}$ and $\mathbf{b}_{x}$ point in the direction of the beam's centerline, while $\mathbf{a}_{y}$ and $\mathbf{a}_{z}$, and $\mathbf{b}_{y}$ and $\mathbf{b}_{z}$ point in the direction of the cross-section's principal axes. For the actual procedure to construct $\mathbf{T}_{\mathrm{e}}$, the reader is referred to the original paper [29]. For an undeformed beam, both sets of nodal basis vectors are equal to the element basis vectors.

In each step of the nonlinear solver, the nodal basis vectors are updated using Rodrigues' rotation formula [31, 32]. For beam end a we get:

$$
\mathbf{T}_{\mathrm{a}}^{t}=\mathbf{R}\left(\Delta \lambda_{\mathrm{a}}\right) \mathbf{T}_{\mathrm{a}}^{t-\Delta t}
$$

where $\mathbf{T}_{\mathrm{a}}^{t}$ collects the current nodal basis vectors, $\mathbf{T}_{\mathrm{a}}^{t-\Delta t}$ collects the nodal basis vectors of the previous iteration, and $\mathbf{R}(\lambda)$ is an orthogonal rotation matrix describing the rotation around a given pseudo-vector $\lambda=\{\alpha \beta \gamma\}^{\mathrm{T}}$ over a given angle $\lambda=\|\lambda\|$, where the double vertical lines denote the Euclidean norm:

$$
\mathbf{R}(\lambda)=\mathbf{I}+\frac{\sin (\lambda)}{\lambda} \mathbf{S}(\lambda)+\frac{1-\cos (\lambda)}{\lambda^{2}} \mathbf{S}(\lambda) \mathbf{S}(\lambda)
$$

where $\mathbf{I}$ is a three-by-three identity matrix, and $\mathbf{S}$ is a skewsymmetric matrix defined as:

$$
\mathbf{S}(\boldsymbol{\lambda})=\left[\begin{array}{ccc}
0 & -\gamma & \beta \\
\gamma & 0 & -\alpha \\
-\beta & \alpha & 0
\end{array}\right]
$$


In equation (9), the vector $\Delta \lambda_{\mathrm{a}}=\left[\Delta \alpha_{\mathrm{a}} \Delta \beta_{\mathrm{a}} \Delta \gamma_{\mathrm{a}}\right]^{\mathrm{T}}$ is the rotation vector describing the change in orientation of beam end a between this iteration and the previous one. In our case, these angle changes are calculated by the implicit dynamic relaxation solver. The updating procedure for the nodal basis vectors of beam end $b$ is analogous.

The deformation of the beam is described by seven independent local beam deformations ${ }^{1}$, recorded at the beam ends. An axial deformation $\delta$ gives rise to a normal force $N$, and six rotational deformations $\theta$ cause six corresponding bending moments $M$. These rotational deformations describe the beam's twist as well as bending around the cross-section's two principal axes at the beam ends.

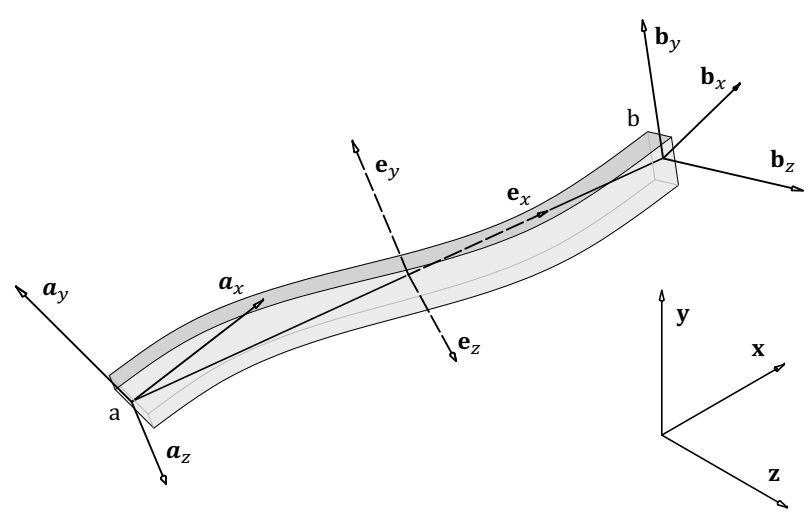

Figure 2: Definition of the nodal basis vectors $\left(\mathbf{a}_{x}, \mathbf{a}_{y}, \mathbf{a}_{z}\right)$ and $\left(\mathbf{b}_{x}, \mathbf{b}_{y}, \mathbf{b}_{z}\right)$ as well as the element basis vectors $\left(\mathbf{e}_{x}, \mathbf{e}_{y}, \mathbf{e}_{z}\right)$ of a deformed beam element.

The local rotational deformations $\theta$ are expressed in terms of the nodal and element basis vectors:

$$
\begin{aligned}
& 2 \sin \left(\theta_{x, \mathrm{a}}\right)=-\left(\mathbf{a}_{z}\right)^{\mathrm{T}} \mathbf{e}_{y}+\left(\mathbf{e}_{z}\right)^{\mathrm{T}} \mathbf{a}_{y} \\
& 2 \sin \left(\theta_{x, \mathrm{~b}}\right)=-\left(\mathbf{b}_{z}\right)^{\mathrm{T}} \mathbf{e}_{y}+\left(\mathbf{e}_{z}\right)^{\mathrm{T}} \mathbf{b}_{y} \\
& 2 \sin \left(\theta_{y, \mathrm{a}}\right)=-\left(\mathbf{a}_{z}\right)^{\mathrm{T}} \mathbf{e}_{x}+\left(\mathbf{e}_{z}\right)^{\mathrm{T}} \mathbf{a}_{x} \\
& 2 \sin \left(\theta_{y, \mathrm{~b}}\right)=-\left(\mathbf{b}_{z}\right)^{\mathrm{T}} \mathbf{e}_{x}+\left(\mathbf{e}_{z}\right)^{\mathrm{T}} \mathbf{b}_{x} \\
& 2 \sin \left(\theta_{z, \mathrm{a}}\right)=-\left(\mathbf{a}_{y}\right)^{\mathrm{T}} \mathbf{e}_{x}+\left(\mathbf{e}_{y}\right)^{\mathrm{T}} \mathbf{a}_{x} \\
& 2 \sin \left(\theta_{z, \mathrm{~b}}\right)=-\left(\mathbf{b}_{y}\right)^{\mathrm{T}} \mathbf{e}_{x}+\left(\mathbf{e}_{y}\right)^{\mathrm{T}} \mathbf{b}_{x}
\end{aligned}
$$

where $\theta_{x}, \theta_{y}$, and $\theta_{z}$ are the deformation angles of the beam ends around the local $x$-, $y$-, and $z$-axis respectively (figure 3 ).

The axial deformation $\delta$ is simply the difference between the current element length $l$ and the undeformed element length $l^{0}$ :

$$
\delta=l-l^{0}
$$

with the length defined as the Euclidian distance between beam ends a and b:

$$
l=\sqrt{\left(x_{\mathrm{a}}-x_{\mathrm{b}}\right)^{2}+\left(y_{\mathrm{a}}-y_{\mathrm{b}}\right)^{2}+\left(z_{\mathrm{a}}-z_{\mathrm{b}}\right)^{2}}
$$

\footnotetext{
${ }^{1}$ Referred to as 'strains' in the original paper. However, because the concerning quantities can not be regarded as strains in the strict mechanical sense, the term 'deformations' is used here instead.
}

where $x_{\mathrm{a}}$ is the $x$-coordinate of node a, $x_{\mathrm{b}}$ is the $x$-coordinate of node $b$, and so on.

The local internal forces are expressed in terms of the local deformations:

$$
\overline{\mathbf{f}}^{e}=\overline{\mathbf{K}}^{e} \overline{\mathbf{u}}^{e}
$$

where $\overline{\mathbf{f}}^{e}$ is the vector collecting all local element forces (figure 3):

$$
\overline{\mathbf{f}}^{e}=\left[\begin{array}{lll}
N & M_{x, \mathrm{a}} & M_{y, \mathrm{a}} M_{z, \mathrm{a}} M_{x, \mathrm{~b}} M_{y, \mathrm{~b}} M_{z, \mathrm{~b}}
\end{array}\right]^{\mathrm{T}}
$$

and $\overline{\mathbf{u}}^{e}$ is the vector collecting all local deformations (figure 3 ):

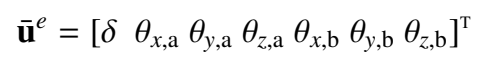

and $\overline{\mathbf{K}}^{e}$ is the local element stiffness matrix, which is based on Euler-Bernoulli beam theory. Furthermore, the material is assumed to be homogeneous, isotropic, and linearly elastic:

$$
\overline{\mathbf{K}}^{e}=\frac{1}{l^{0}}\left[\begin{array}{ccccccc}
E A & 0 & 0 & 0 & 0 & 0 & 0 \\
0 & G J & 0 & 0 & -G J & 0 & 0 \\
0 & 0 & 4 E I_{y} & 0 & 0 & 2 E I_{y} & 0 \\
0 & 0 & 0 & 4 E I_{z} & 0 & 0 & 2 E I_{z} \\
0 & -G J & 0 & 0 & G J & 0 & 0 \\
0 & 0 & 2 E I_{y} & 0 & 0 & 4 E I_{y} & 0 \\
0 & 0 & 0 & 2 E I_{z} & 0 & 0 & 4 E I_{z}
\end{array}\right]
$$

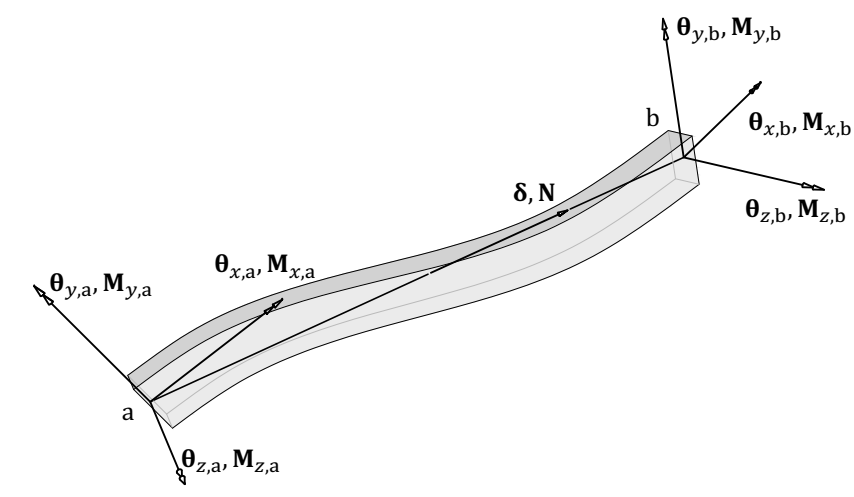

Figure 3: Orientation of the local beam deformations $\left(\begin{array}{lllllll}\delta & \theta_{x, \mathrm{a}} & \theta_{y, \mathrm{a}} & \theta_{z, \mathrm{a}} & \theta_{x, \mathrm{~b}} & \theta_{y, \mathrm{~b}} & \theta_{z, \mathrm{~b}}\end{array}\right)$ and corresponding internal forces $\left(N M_{x, \mathrm{a}} M_{y, \mathrm{a}} M_{z, \mathrm{a}} M_{x, \mathrm{~b}} M_{y, \mathrm{~b}} M_{z, \mathrm{~b}}\right)$.

The global element forces $\mathbf{f}^{e}$ are related to the local forces $\overline{\mathbf{f}}^{e}$ as follows:

$$
\mathbf{f}^{e}=\mathbf{T}^{e} \overline{\mathbf{f}}^{e}
$$

where $\mathbf{f}^{e}=\left[f_{x, \mathrm{a}} f_{y, \mathrm{a}} f_{z, \mathrm{a}} m_{x, \mathrm{a}} m_{y, \mathrm{a}} m_{z, \mathrm{a}} f_{x, \mathrm{~b}} f_{y, \mathrm{~b}} f_{z, \mathrm{~b}} m_{x, \mathrm{~b}} m_{y, \mathrm{~b}} m_{z, \mathrm{~b}}\right]^{\mathrm{T}}$ is the vector containing forces and moments in the global $x-, y$-, and $z$-axis for beam end a and beam end $\mathrm{b}$, and $\mathbf{T}^{e}$ is a transformation matrix defined as

$$
\mathbf{T}^{e}=\left(\frac{\partial \overline{\mathbf{u}}^{e}}{\partial \mathbf{x}^{e}}\right)^{\mathrm{T}}
$$


where $\mathbf{x}^{e}=\left[\begin{array}{llllllllllll}x_{\mathrm{a}} & y_{\mathrm{a}} & z_{\mathrm{a}} & \alpha_{\mathrm{a}} & \beta_{\mathrm{a}} & \gamma_{\mathrm{a}} & x_{\mathrm{b}} & y_{\mathrm{b}} & z_{\mathrm{b}} & \alpha_{\mathrm{b}} & \beta_{\mathrm{b}} & \gamma_{\mathrm{b}}\end{array}\right]^{\mathrm{T}}$ is a vector collecting translational and rotational coordinates of beam end $\mathrm{a}$ and beam end $\mathrm{b}$ in the global $x$-, $y$-, and $z$-direction. For the actual calculation of the transformation matrix $\mathbf{T}^{e}$, the reader is referred to the original paper [29]. The full internal force vector $\mathbf{f}$ is assembled from the element forces $\mathbf{f}^{e}$, with $e=1 \ldots m$, where $m$ is the number of elements.

The element tangential stiffness matrix is obtained from differentiation of the internal forces $\mathbf{f}^{e}$ (equation (19)) with respect to the element position vector $\mathbf{x}^{e}$ :

$$
\begin{aligned}
\mathbf{K}^{e} & =\frac{\mathrm{d} \mathbf{f}^{e}}{\mathrm{~d} \mathbf{x}^{e}}=\frac{\partial \mathbf{f}^{e}}{\partial \overline{\mathbf{f}}^{e}} \frac{\partial \overline{\mathbf{f}}^{e}}{\partial \overline{\mathbf{u}}^{e}} \frac{\mathrm{d} \overline{\mathbf{u}}^{e}}{\mathrm{~d} \mathbf{x}^{e}}+\frac{\partial \mathbf{f}^{e}}{\partial \mathbf{T}^{e}} \frac{\mathrm{d} \mathbf{T}^{e}}{\mathrm{~d} \mathbf{x}^{e}} \\
& =\mathbf{T}^{e} \overline{\mathbf{K}}^{e} \mathbf{T}^{e^{\mathrm{T}}}+\mathbf{K}_{\mathrm{g}}^{e}=\mathbf{K}_{\mathrm{e}}^{e}+\mathbf{K}_{\mathrm{g}}^{e}
\end{aligned}
$$

The resulting stiffness matrix consists of an elastic part $\mathbf{K}_{\mathrm{e}}^{e}$ and a geometric part $\mathbf{K}_{\mathrm{g}}^{e}$. The derivation of the geometric stiffness matrix $\mathbf{K}_{\mathrm{g}}^{e}$ can be found in the original paper [29]. The element stiffness matrix $\mathbf{K}_{i}^{e}$ for each element $i=1 \ldots m$ is used to assemble the full tangential stiffness matrix $\mathbf{K}$.

\section{Prototype}

In order to validate the proposed design framework, and to test the proposed way of controlling the gridshell's shape by changing the lengths of the cables, a scale model has been designed and built. The gridshell model has a footprint of around $0.9 \mathrm{~m}$ by $0.6 \mathrm{~m}$. Although the model was designed as an independent structure on scale $1: 1$, it can be regarded as a 1 to 20 scale representation of an actual gridshell. The following subsection (4.1) describes the design of the model. Its construction is discussed in subsection 4.2.

The purpose of this prototype example it to show the possible challenges that can be encountered during the design and construction of a gridshell serving as the formwork of a concrete shell, and demonstrate how the methodology proposed in this paper can be used to overcome these issues. Therefore, design choices are made specifically to uncover possible problems. It is not our aim to make an exhaustive design and analysis fulfilling all relevant norms.

\subsection{Design}

As a preliminary step, the input parameters for the design framework were determined. A concrete shell was designed using RhinoVault [33], which is a plug-in for Rhino to design funicular shells. The plugin is based on the Thrust Network Analysis method, developed by Block and Ochsendorf [34]. The 3D form diagram, and its corresponding 2D form and force diagrams are shown in figure 4 . Although the envisioned shell has a few openings, we decided that the supporting gridshell had to rest completely on the ground. Therefore, the openings in the shell were closed to define the actual target shape for the gridshell falsework (figure 5).

Requirements regarding the workability of the grid rods and their availability in the required scale limited the options for the rods' cross-sections. Therefore, the cross-section of the rods was chosen beforehand, implying that only the undeformed
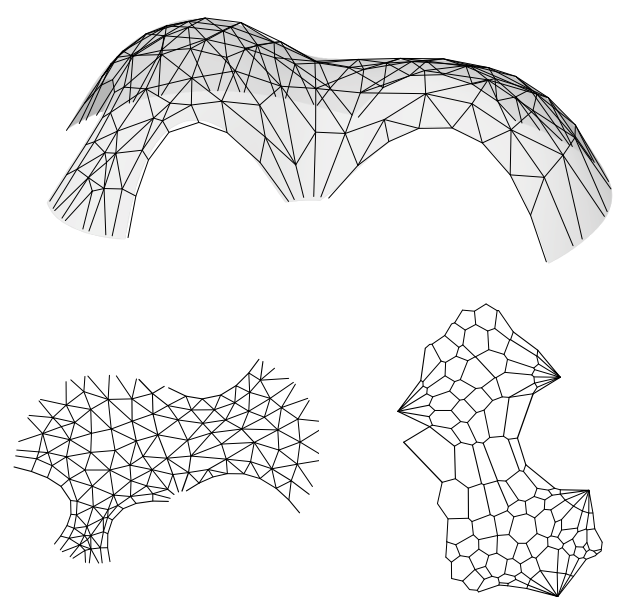

Figure 4: A funicular shell is designed using RhinoVault, where the twodimensional form (left) and force (right) diagrams are manipulated to alter the three-dimensional force network (top).

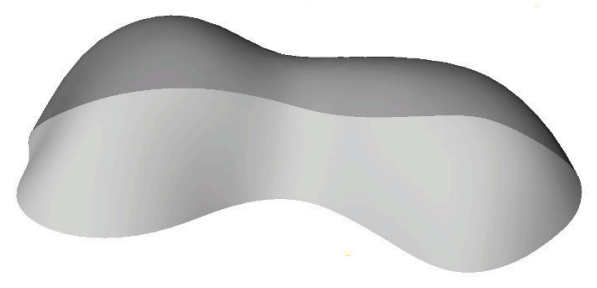

Figure 5: Openings in the shell design were closed to define the target shape for the supporting gridshell. 
lengths of the bracing cables were selected as design variables, and the fourth step of the design framework (figure 1) was omitted. The chosen rods have a diameter of $d=2 \mathrm{~mm}$, and are made of glass-fibre reinforced polymer, with a Young's modulus of $40 \mathrm{GPa}$. Each rod segment spanning between two connections was modeled with four co-rotational beam elements to capture potential local beam buckling with sufficient accuracy, while keeping the required computation time acceptable. The spacing between grid rods was set to $55 \mathrm{~mm}$. The supports were modeled as pinned. The bracing elements were modeled as single cable elements with an axial stiffness of $E A=187$ $\mathrm{N}$, based on preliminary tests. Finally, the connections between grid rods were modeled as short beam elements perpendicular to the gridshell's surface, simulating the eccentricity between crossing rods.

The structural properties of the connections were initially guessed. However, it was observed that they had a significant influence on the structural behavior of the model. Therefore, in a second stage, they were calibrated based on data from a test on the physical model, before bracing was applied. The test data consisted of two 3D scans: one without loading and one with point loads of $300 \mathrm{~g}$ on the locations shown in figure 9. The scans measured the location of 42 nodes distributed over the gridshell using a Vicon motion capture setup [35]. To calibrate the structural parameters of the connections, an inverse problem had to be solved, which was achieved by slightly adapting the numerical optimization approach described above: the structural parameters of the connections were selected as design variables to minimize the discrepancy between predictions by the numerical model and data obtained from the test on the physical model. The resulting parameters are: $E A=7.15 \times 10^{4}$ $\mathrm{N}, G J=7.38 \times 10^{-4} \mathrm{Nm}^{2}, E I_{y}=E I_{z}=7.85 \times 10^{-4} \mathrm{Nm}^{2}$. These parameters were validated by comparing predictions and measurements for an additional load case. For a large-scale gridshell, we suggest to determine the connection properties by means of experiments on a full-scale mockup structure consisting of at least two crossing bars.

A comparison between the grid properties of the scale model and an equivalent large-scale gridshell is shown in table 1 to more easily interpret the dimensions and loads in the model. The approximated equivalent point load per connection node is $3.7 \mathrm{~N}$ for the chosen grid spacing, corresponding to $5 \mathrm{~cm}$ of concrete, assuming a square tributary area for each load. However, for practical reasons, loads are only applied every 2 connection nodes. Consequently, the weight of all loads was set to $700 \mathrm{~g}$ after rounding and compensating for the fact that the tributary areas are no longer perfectly square for the erected gridshell.

\section{Generation of target grid}

Next, a target grid was generated by pulling an oversized flat grid towards the surface using implicit dynamic relaxation to simulate the bending process. The grid was forced on the surface by assigning a force density to a series of fictitious springs that were allowed to slide over the surface. After a predefined number of iterations of the nonlinear solver, the oversized grid fitted the target surface, and excess parts were trimmed (figure 6 ). The corresponding initial, flat grid (figure 7) and the posi- tions of the supports (figure 8) were derived from the trimmed grid. Moreover, the maximum allowable radius for the crosssection of the grid rods was found to be $1.3 \mathrm{~mm}$ using equation (1). This is larger than the cross-section we chose, meaning that the chosen cross-section is feasible.

\section{Generation of initial design}

Next, the initial design for the optimization was generated by assigning the actual physical properties to all elements in the finite element model used in the previous step. Although the flowchart (figure 1) suggests to assign the maximum allowable cross-section, the cross-section chosen previously is assigned instead, as it is not considered as a design variable in this case. Cables are placed on every feasible location, excluding cables that would have to be connected to the supports of the gridshell and cables that would rise above the shell surface, as these would hinder the application of the concrete. The undeformed lengths of the cables were initially set to $99 \%$ of the distances between the nodes they connect in the target grid, determined in the previous step. The initial design was subjected to point loads of $700 \mathrm{~g}(6.9 \mathrm{~N})$, placed on every 2 nodes in the area that would be covered by the actual shell (figure 9). Furthermore, the initial design was subjected to an additional load case of 1.2 times the design load in order to constrain the deformations from this additional load case in the following optimization step. If the initial design deforms too heavily under these load conditions, it is too far from its optimum for the optimization algorithm to converge. Figures 12b, 13b, and 14b show the unloaded initial design, the initial design loaded with $6.9 \mathrm{~N}$ per two connection nodes, and the initial design loaded with $8.2 \mathrm{~N}$ per two connection nodes, respectively, where the colors indicate the distance to the target grid. Although deviations from the target shape are large for the additional load case, they are still manageable for the optimizer, and, consequently, we can proceed to the next step.

\section{Optimization of undeformed cable lengths}

In this step, the undeformed lengths of the cables are optimized according to the optimization approach discussed in section 2. The weighting factors are chosen so that the value of the objective function (2) is 1 for the initial design, and the first and second term respectively initially contribute $70 \%$ and 30 $\%$ to make sure the optimized design fits the target sufficiently well, while reducing the number of cables as much as possible. Because only the undeformed cable lengths are chosen as the design variables for this case, the last term in equation (2), minimizing the cross-section of the rods, is excluded by setting the corresponding weighting factor $w_{3}$ to zero. The resulting values for the other two weighting factors are: $w_{1}=41 \mathrm{~m}^{-2}$ and $w_{2}=5.3 \times 10^{-4} \mathrm{~N}^{-1}$. Constraints (3), (5), and (6) were taken into account, where each value of $\mathbf{l}_{\mathrm{c}, \text { min }}^{0}$ was set to $1 \mathrm{~cm}$, $\mathbf{l}_{\mathrm{c}, \max }^{0}$ was set to 1.1 times the initial undeformed cable lengths, each value of $\mathbf{n}_{\max }$ was set to $20 \mathrm{~N}$, constraint (6) considered an additional load case of 1.2 times the design load, and $d^{2}$ was set to $0.037 \mathrm{~m}^{2}$, which corresponds to a RMS deviation from the target of $5 \mathrm{~mm}$ per node. 


\begin{tabular}{l|cc} 
& scale model & equivalent gridshell \\
\hline length scale & $1 / 1$ & $20 / 1$ \\
weight scale & $1 / 1$ & $400 / 1$ \\
\hline gridshell length & $0.90 \mathrm{~m}$ & $18.0 \mathrm{~m}$ \\
gridshell width & $0.55 \mathrm{~m}$ & $11.0 \mathrm{~m}$ \\
gridshell height & $0.21 \mathrm{~m}$ & $4.2 \mathrm{~m}$ \\
distance between rods & $0.055 \mathrm{~m}$ & $1.1 \mathrm{~m}$ \\
radius of cross-section of rods & $0.001 \mathrm{~m}$ & $0.02 \mathrm{~m}$ \\
shell thickness & $0.0025 \mathrm{~m}$ & $0.05 \mathrm{~m}$ \\
density of concrete & $50000 \mathrm{~kg} / \mathrm{m}^{3}$ & $2500 \mathrm{~kg} / \mathrm{m}^{3}$ \\
distributed load & $1.2 \times 10^{3} \mathrm{~N} / \mathrm{m}^{2}$ & $1.2 \times 10^{3} \mathrm{~N} / \mathrm{m}^{2}$ \\
equivalent point load per connection node & $3.7 \mathrm{~N}$ & $1.5 \times 10^{3} \mathrm{~N}$
\end{tabular}

* Approximation assuming a square tributary area.

The method of moving asymptotes [36] was chosen as the optimization algorithm. The convergence history is shown in figure 10 for the objective function (equation (2)), which shows a reduction from 1 to 0.54 in only 49 iterations, as both terms in the objective function decreased. As a result, the optimized gridshell fits the target better than the initial design, while requiring only 130 cables instead of the 293 in the initial design. The convergence history of the constraints is given in figure 11, showing $\left(\mathbf{d}_{1}^{\mathrm{T}} / \mathbf{d}_{1}\right) / d^{2}-1$ and the maximum value of $\mathbf{n}_{\mathrm{c}} / \mathbf{n}_{\mathrm{c}, \max }-1$ for each iteration, such that negative values indicate a feasible design. The constraint on the normal forces in the cables does not become active, as the normal forces do not exceed $12.0 \mathrm{~N}$ during the optimization. On the other hand, the constraint limiting the deviation from the target grid under additional loading is heavily violated in the initial design. The optimizer is capable of reducing this deviation until the design is feasible. The deviation of the optimized gridshell from the target grid is shown in figure $13 \mathrm{~d}$ for the design load, and figure $14 \mathrm{~d}$ for the additional load case.

To show the importance of taking the applied loads into account, an optimized design where the loads were neglected during optimization is also considered. One could argue that this design is achievable with currently available design methods, by deriving the ideal (deformed) cable lengths from the target grid, and using this information to iteratively tension the cables in the physical gridshell until their lengths match the ideal lengths, before applying the final loads. Such a design is compared to the design optimized according to the procedures described previously, as well as the initial design and an unbraced design in figures 12, 13, 14 for the three following cases: without loads, under the design load case, and under the additional load case.

Comparison of the unloaded designs (figure 12) shows that the unbraced gridshell does not fit the target shape very well, with deviations exceeding $1 \mathrm{~cm}$ in a large area of the gridshell. This shows the need for a system to control the shape of the relaxed gridshell, such as the bracing cables with adaptive lengths suggested in this paper. Comparison of the loaded designs (figure 13) shows that some type of bracing system is required anyway, as the unbraced gridshell completely snaps through under the design load. Although the initial design and the optimized design which does not consider the wet concrete loading fit the target grid very well without loads (figure 12), they perform much worse than the optimized design once the design load is applied (figure 13). Furthermore, they are much more sensitive to an increased loading, despite having more than twice as many cables: figure 14 shows detrimental deformations for all designs but the optimized design for the additional load case of 1.2 times the design load.

In order to assess the impact of fixing the rod diameter in advance, and to demonstrate the full optimization approach, we perform the optimization a second time, now disregarding the constraint on the rod diameter. The maximum allowable rod radius of $1.3 \mathrm{~mm}$ is used as initial value. The weighting factors in the objective function are now chosen so that, for the initial design, the first, second, and third term in the objective function equal $0.6,0.2$, and 0.2 , respectively. This results in the following values: $w_{1}=70 \mathrm{~m}^{-2}, w_{2}=3.05 \times 10^{-4} \mathrm{~N}^{-1}, w_{3}=154$ $\mathrm{m}^{-1}$. The weighting factors in this example are set to balance the effect of all parts of the objective function to demonstrate the potential and limitations of the approach. In a real application, the first part of the objective function would typically be most important.

The optimization problem is solved in 49 iterations. Figures 15 and 16 show the convergence history of the objective function and the constraints, respectively. The final value of the rod radius is $1.12 \mathrm{~mm}$, which is slightly more than the fixed value of $1 \mathrm{~mm}$ considered before. Dropping the constraint on the rod radius results in a larger design space, which should give rise to a better optimum: the optimized design includes 86 cables, which is less than the 130 cables obtained before. The misfit $\mathbf{d}^{\mathrm{T}} \mathbf{d}$ between the final shape and the target shape of the shell is $44 \%$ smaller than before, and the L1 norm $\sum_{i=1}^{n_{\mathrm{c}}} N_{i}$ of the cable forces is $54 \%$ smaller. These gains are achieved at the expense 

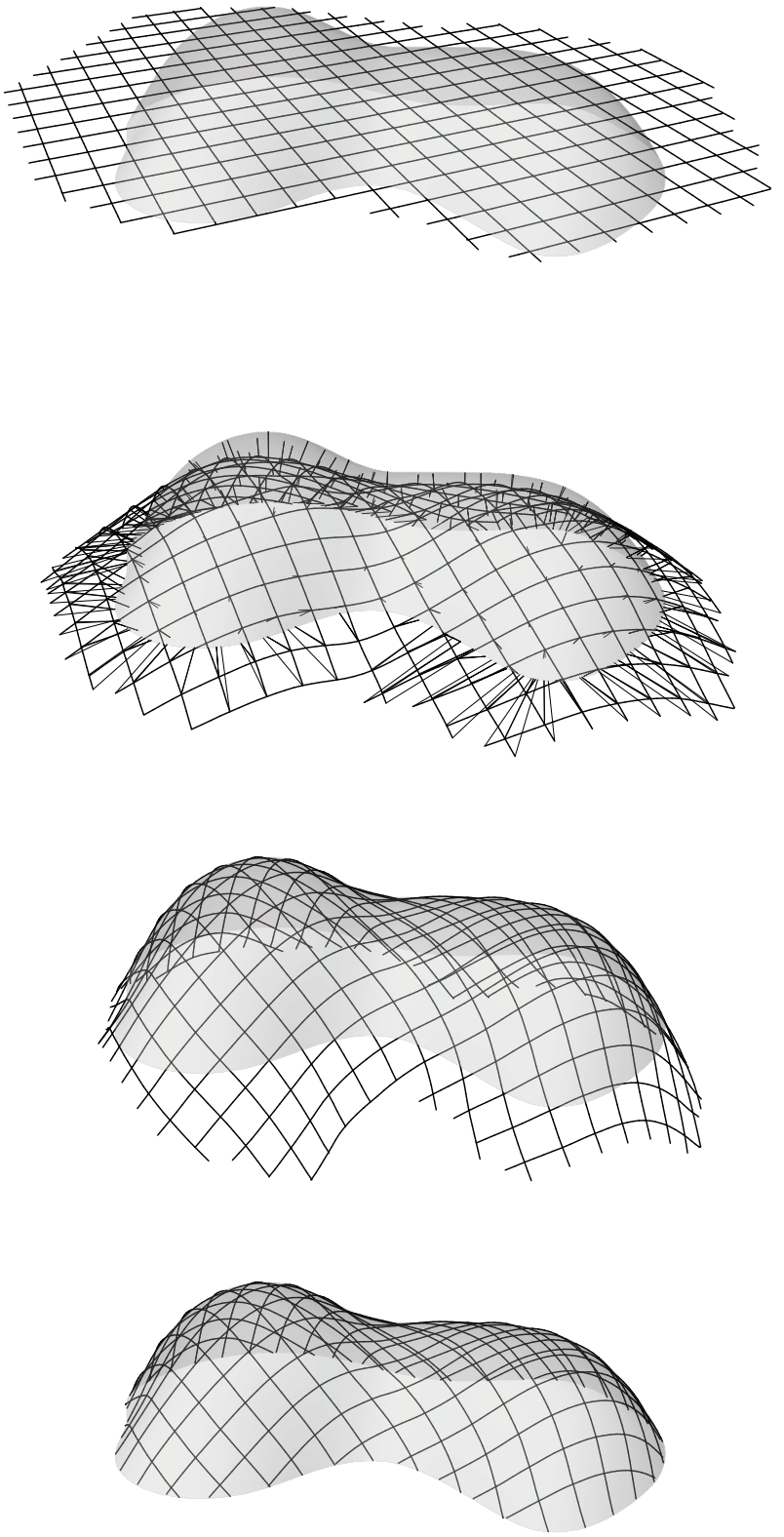

Figure 6: A target grid is generated by pulling an oversized flat grid towards the target surface and trimming away excess parts.

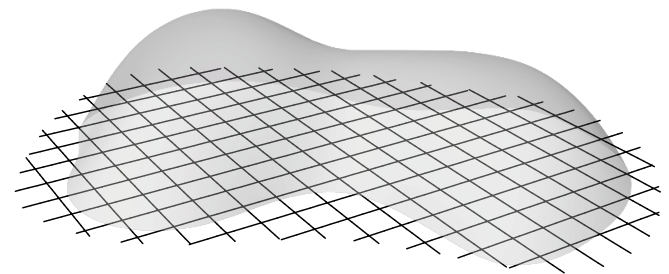

Figure 7: The initial, flat grid is derived from the target grid.

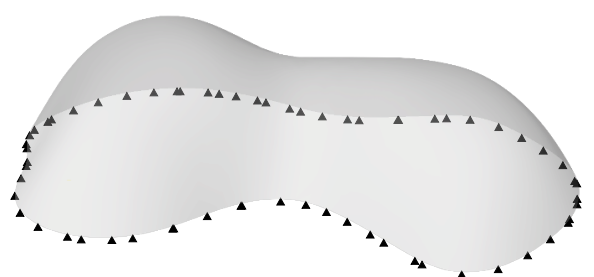

Figure 8: The locations of the supports is derived from the target grid.

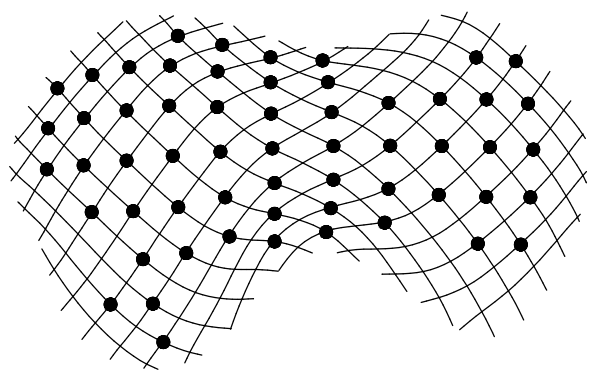

Figure 9: Top view. Loads (black dots) are applied every two nodes in the area covered with concrete. 


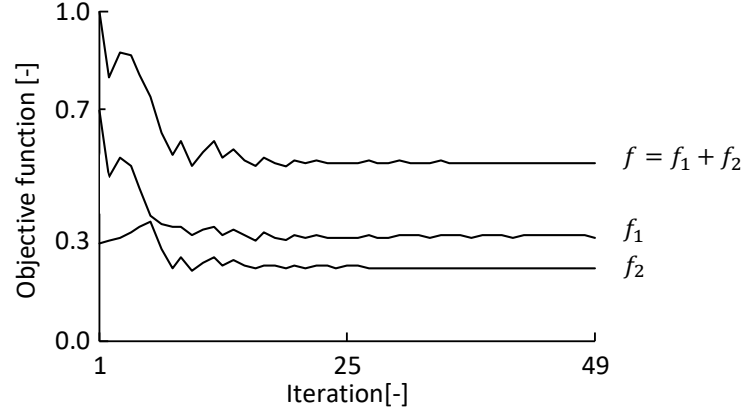

Figure 10: Convergence history of the objective function for a fixed rod diameter of $2 \mathrm{~mm}$. The optimizer reduces the objective function $f$ from 1 to 0.54 in 49 iterations by reducing both terms $f_{1}=w_{1} \mathbf{d}^{\mathrm{T}} \mathbf{d}$ and $f_{2}=w_{2} \sum_{i=1}^{n_{\mathrm{c}}} N_{i}$.

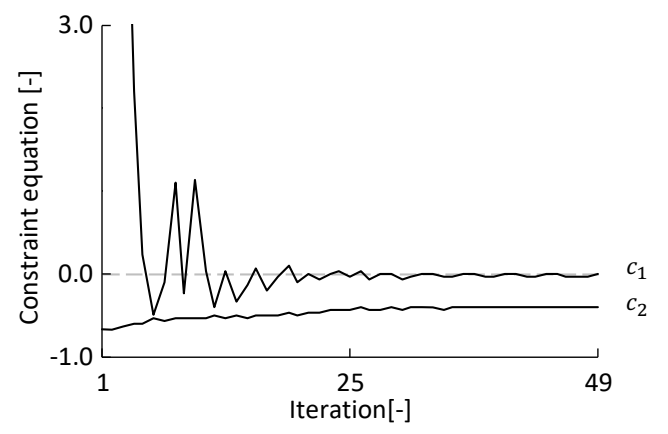

Figure 11: Feasibility of the design with a fixed rod diameter of $2 \mathrm{~mm}$ during optimization. $c_{1}=\left(\mathbf{d}_{1}^{\mathrm{T}} / \mathbf{d}_{1}\right) / d^{2}-1$ is reduced from 23.4 to 0.0 , while $c_{2}=$ $\mathbf{n}_{\mathrm{c}} / \mathbf{n}_{\mathrm{c}, \max }-1$ increases from -0.7 to -0.4 , which means that the optimized design is feasible. of the rod radius $r_{\mathrm{g}}$, which is $12 \%$ larger than before. If this is not desirable, the optimization problem has to be solved again with different values for the weighting terms in the objective function.

\subsection{Construction}

In this subsection, the construction of the scale model designed above is discussed. The construction went through several stages. First, the flat grid was assembled by tying the individual rods together with a square lash using an elastic band to approximate cylindrical hinges. Sliding of the knot was further restricted by applying a thin strip of duct-tape next to the knot. The result (figure 18) is a connection that is relatively flexible for rotations in the plane of the grid, and stiff with respect to other deformations. Subsequently, the grid was erected by putting each rod end in a superficial socket, with a thin hole running through the support plate to add a tension cable (figure 17). The resulting support is able to generate reaction forces in almost any direction.

Figure 20 shows the gridshell's geometry at this stage, and a comparison between the physical and numerical model is made by visually superimposing the numerical model on the physical model. Additionally, the geometry is captured by scanning several marked nodes on the model. The scan is performed using a Vicon motion capture setup [35] and 42 spherical markers distributed over the gridshell. An average error of $0.15 \mathrm{~mm}$ and a variability lower than $0.025 \mathrm{~mm}$ have been reported for static experiments [37]. However, due to slightly inaccurate placement of the markers, the maximum error of the results is expected to be around $2 \mathrm{~mm}$. The distance between the scanned nodes and the modeled nodes is $3.1 \mathrm{~mm}$ on average, and at most $7.4 \mathrm{~mm}$ (table 2). Note that the maximum difference between the numerical model and the physical model at this stage is equal to the maximal deviation from the target of the optimized numerical model. In other words, for the current level of accuracy, it would be pointless to further minimize the predicted deviation from the target.

To further check the accuracy of the finite element model, the scale model is subjected to two additional load cases before adding the bracing cables. The first load case consists of point loads of $300 \mathrm{~g}$ acting on the same nodes as the design load case (figure 9). The scale model is compared to the finite element model under these loading conditions in figure 21 . Another scan showed an average distance of $4.0 \mathrm{~mm}$, and maximum distance of $8.8 \mathrm{~mm}$ between the physical model and the numerical model (table 2). The second load case consists of 10 point loads of $500 \mathrm{~g}$ acting in the middle of the gridshell (figure 19). The distance between the numerical model and the physical model ranges up to $8.0 \mathrm{~mm}$, with an average of $3.2 \mathrm{~mm}$ (figure 22, table 2).

In the next stage, bracing cables were added to the scale model in the optimized locations. The bracing consists of two double nylon wire strings, and a tensioning mechanism composed of 2 cable ties (figure 23). Once all cables were placed, their undeformed lengths were set to their optimized values by tensioning the cable ties. Because we had no way of measuring the forces in the cables, the cables were tensioned based only on 


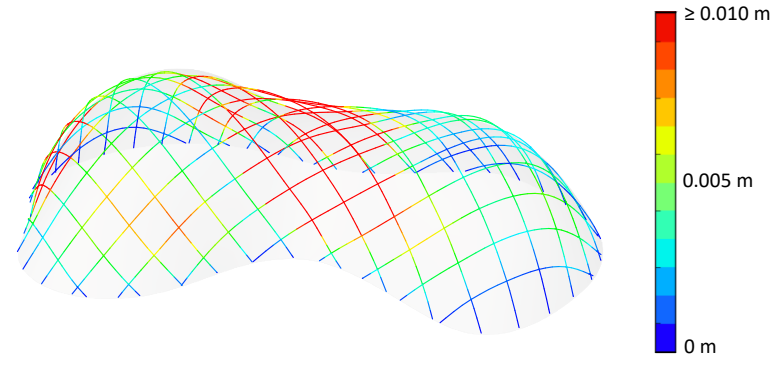

(a) Unbraced design

( maximum deviation $=44.9 \mathrm{~mm}$, average deviation $=6.7 \mathrm{~mm}$ )

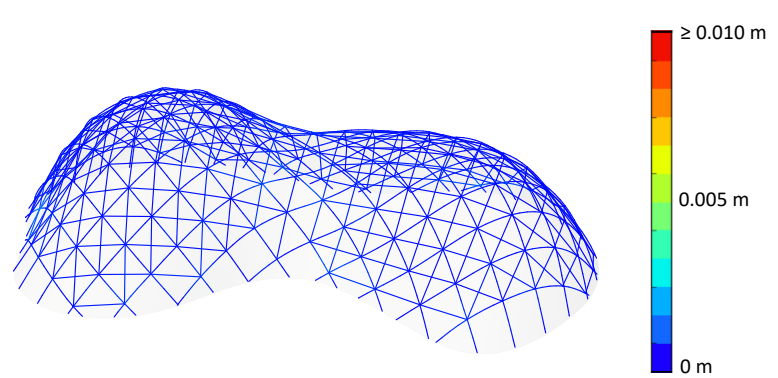

(c) Optimized design, without consideration of loads

$($ maximum deviation $=1.3 \mathrm{~mm}$, average deviation $=0.2 \mathrm{~mm})$

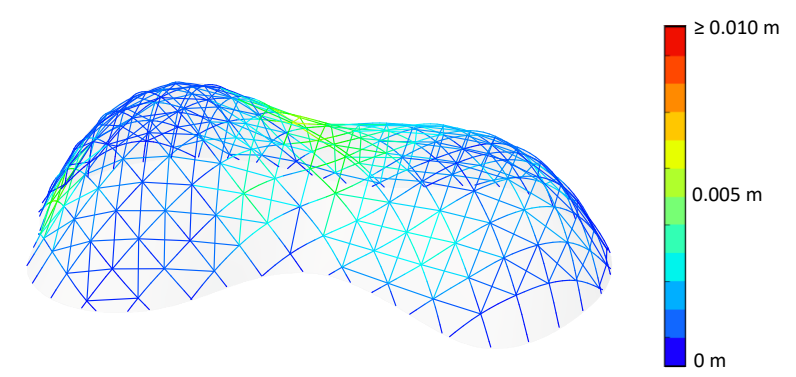

(b) Initial design

(maximum deviation $=7.4 \mathrm{~mm}$, average deviation $=1.6 \mathrm{~mm}$ )

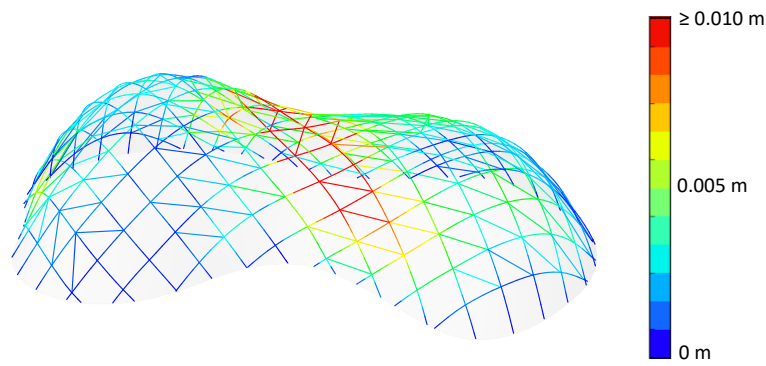

(d) Optimized design

(maximum deviation $=15.0 \mathrm{~mm}$, average deviation $=3.4 \mathrm{~mm})$

Figure 12: Deviation from target grid (indicated in color) without loads.

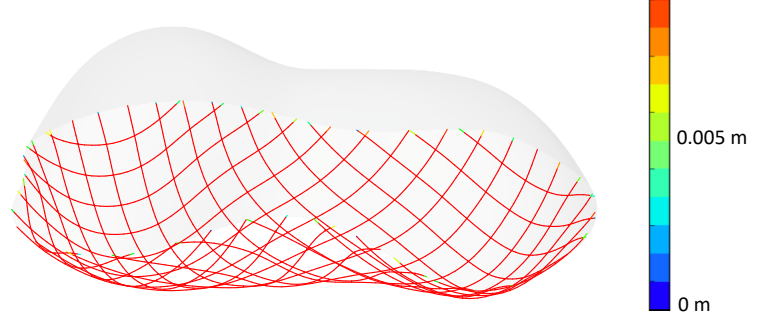

(a) Unbraced design

$($ maximum deviation $=416.7 \mathrm{~mm}$, average deviation $=191.3 \mathrm{~mm})$

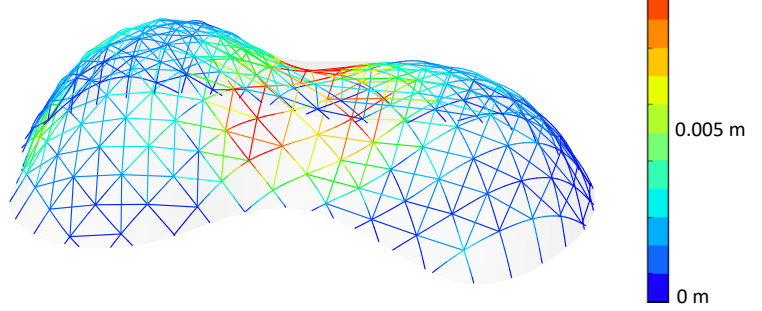

(b) Initial design

( maximum deviation $=14.5 \mathrm{~mm}$, average deviation $=2.6 \mathrm{~mm}$ )

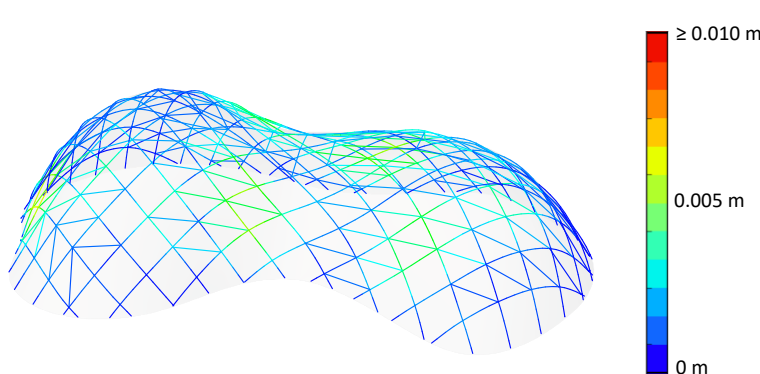

(d) Optimized design

$($ maximum deviation $=7.4 \mathrm{~mm}$, average deviation $=1.8 \mathrm{~mm})$

(c) Optimized design, without consideration of loads

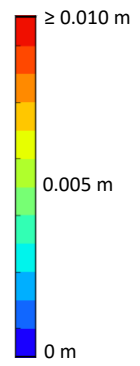

Figure 13: Deviation from target grid (indicated in color) under the design load case. 


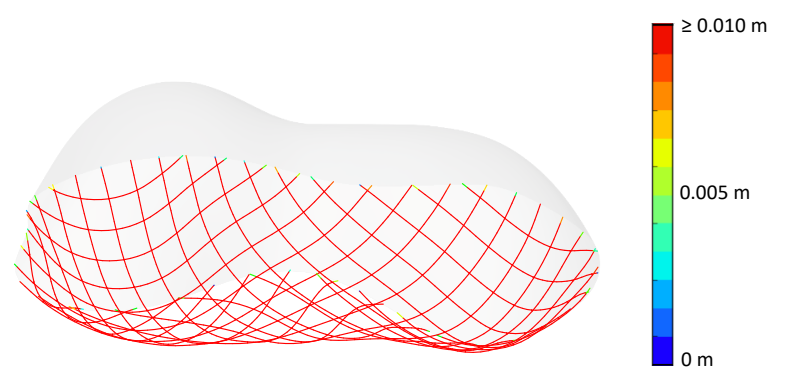

(a) Unbraced design

$($ maximum deviation $=414.5 \mathrm{~mm}$, average deviation $=193.0 \mathrm{~mm})$

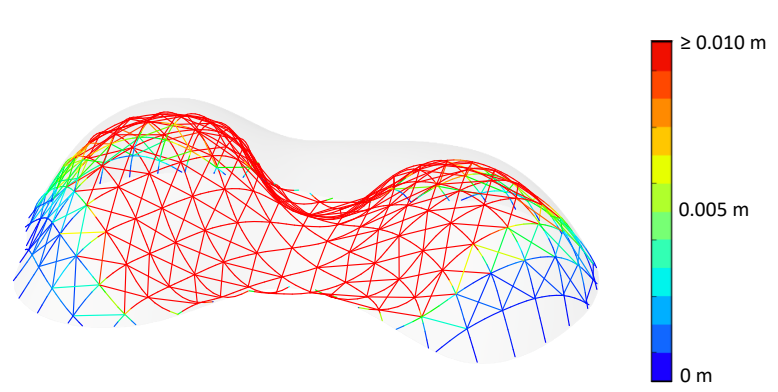

(c) Optimized design, without consideration of loads

(maximum deviation $=138.5 \mathrm{~mm}$, average deviation $=37.2 \mathrm{~mm})$

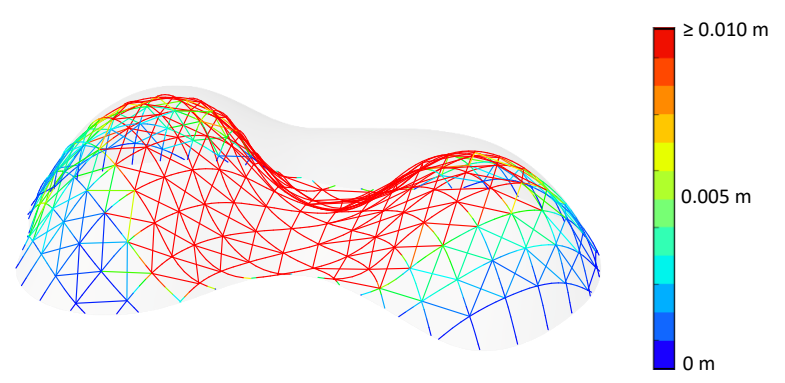

(b) Initial design

(maximum deviation $=133.0 \mathrm{~mm}$, average deviation $=30.2 \mathrm{~mm})$

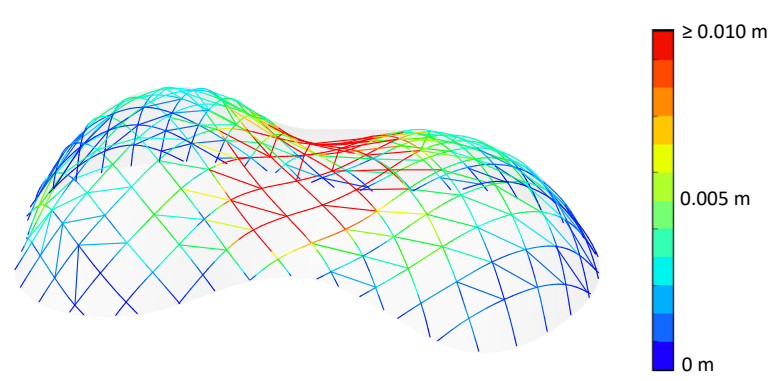

(d) Optimized design

$($ maximum deviation $=23.1 \mathrm{~mm}$, average deviation $=4.1 \mathrm{~mm})$

Figure 14: Deviation from target grid (indicated in color) under the additional load case.

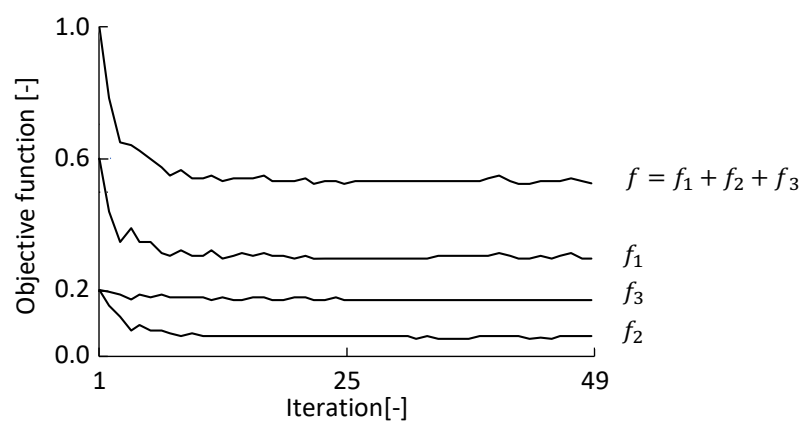

Figure 15: Convergence history of the objective function for a variable rod diameter. The optimizer reduces the objective function $f$ from 1 to 0.52 in 49 iterations by reducing the terms $f_{1}=w_{1} \mathbf{d}^{\mathrm{T}} \mathbf{d}, f_{2}=w_{2} \sum_{i=1}^{n_{\mathrm{c}}} N_{i}$, and $f_{3}=w_{3} r_{\mathrm{g}}$.

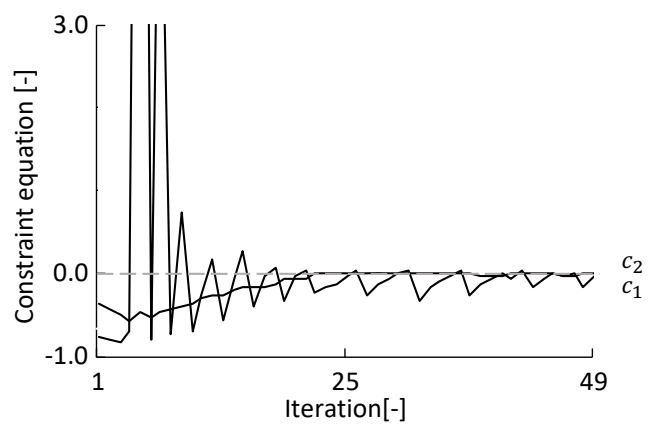

Figure 16: Feasibility of the design with a variable rod diameter during optimization. $\quad c_{1}=\left(\mathbf{d}_{1}^{\mathrm{T}} / \mathbf{d}_{1}\right) / d^{2}-1$ increases from -0.78 to -0.05 , and $c_{2}=$ $\mathbf{n}_{\mathrm{c}} / \mathbf{n}_{\mathrm{c}, \max }-1$ increases from -0.37 to -0.01 , which means that the optimized design is feasible. 


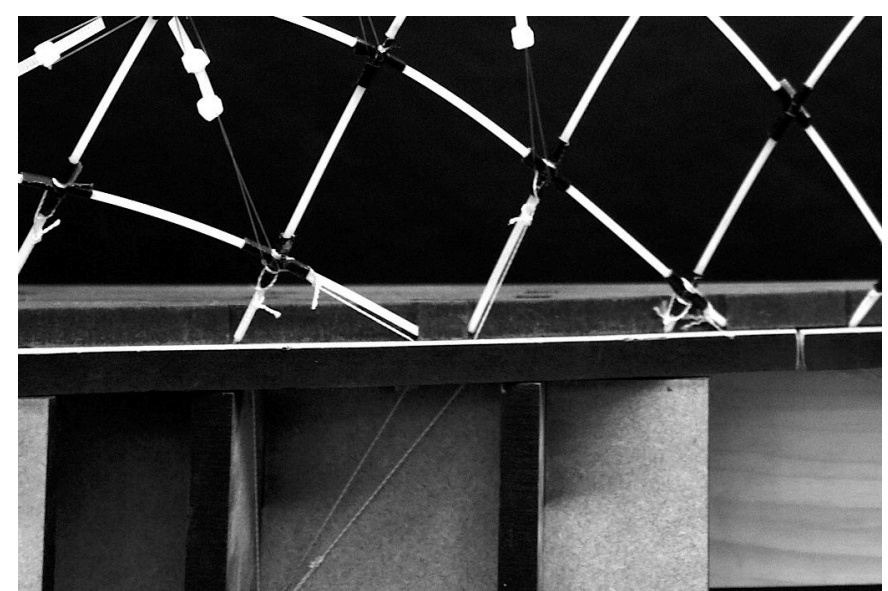

Figure 17: The supports in the physical model consist of a small socket and a tension cable running through the support plate.

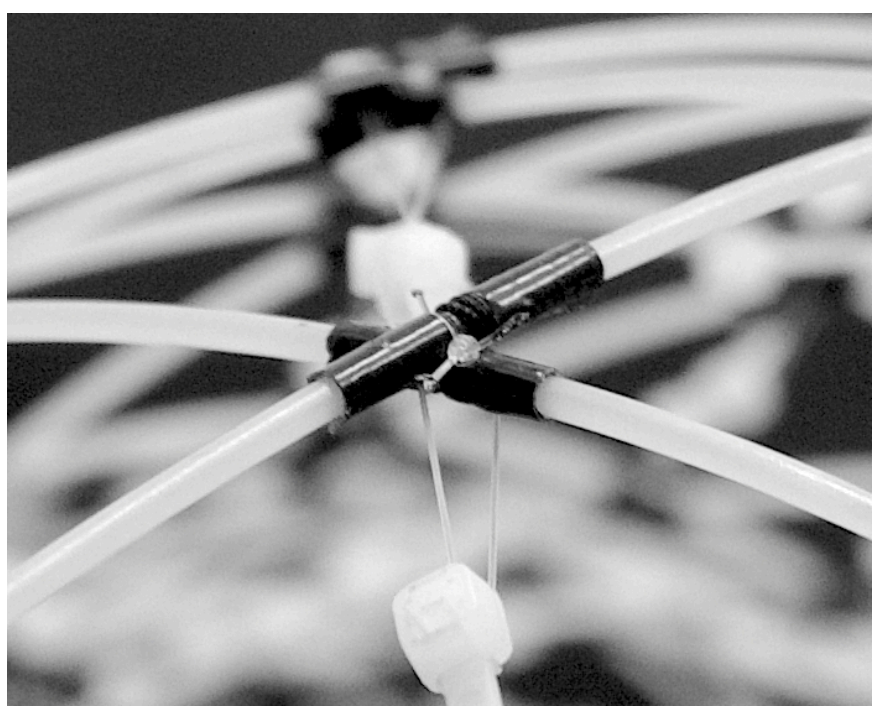

Figure 18: Rod connections are made using an elastic nylon band with ducttape on the sides to restrict sliding of the knot.

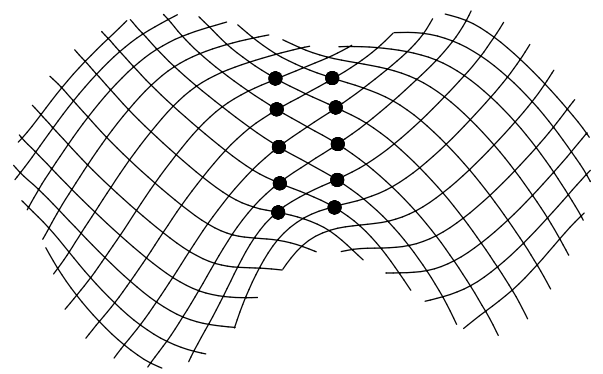

Figure 19: Top view. Ten point loads (black dots) are applied in the middle of the gridshell.
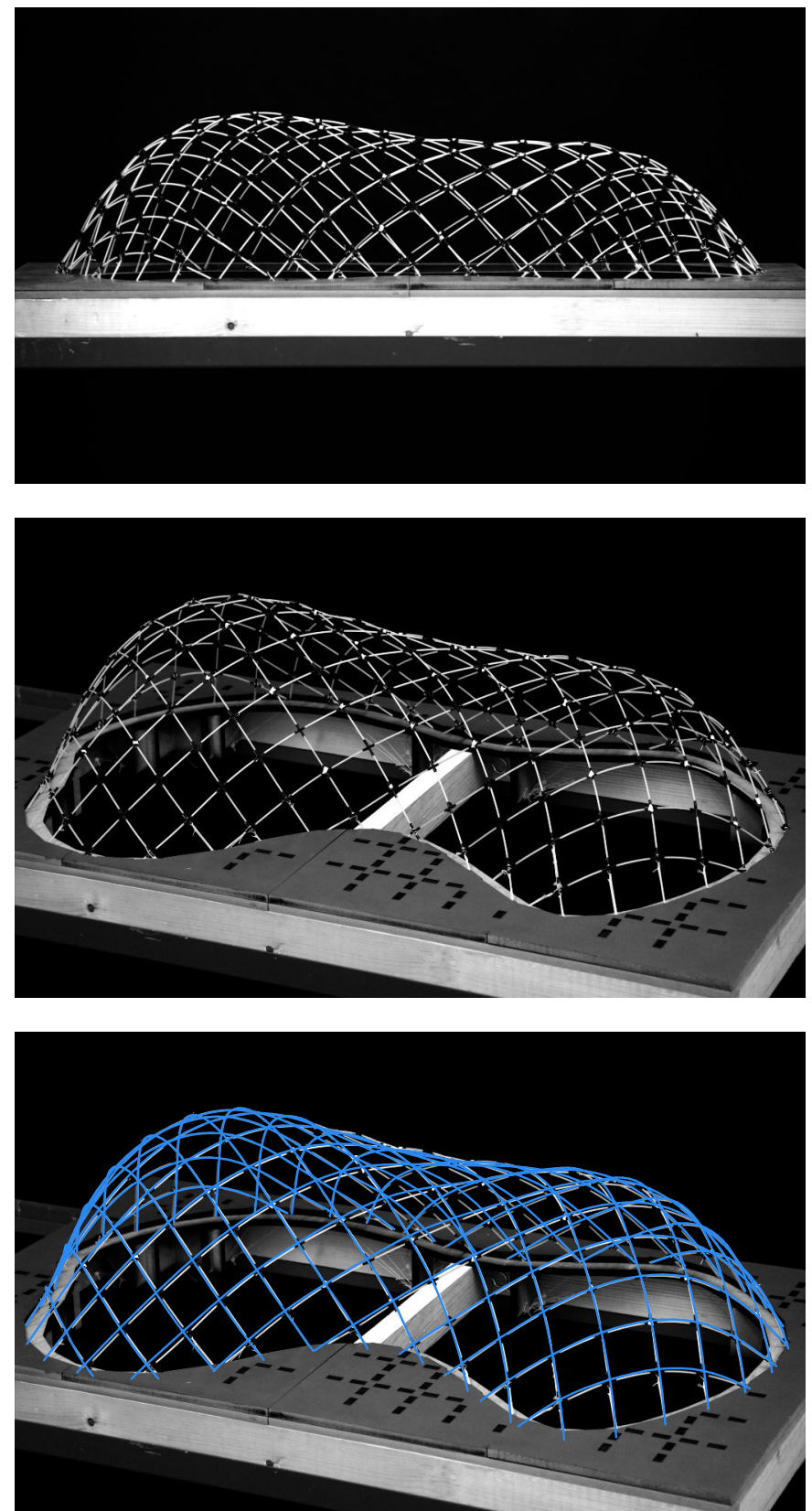

Figure 20: Geometry of the physical model without braces and without loads, with the corresponding numerical model in blue. 

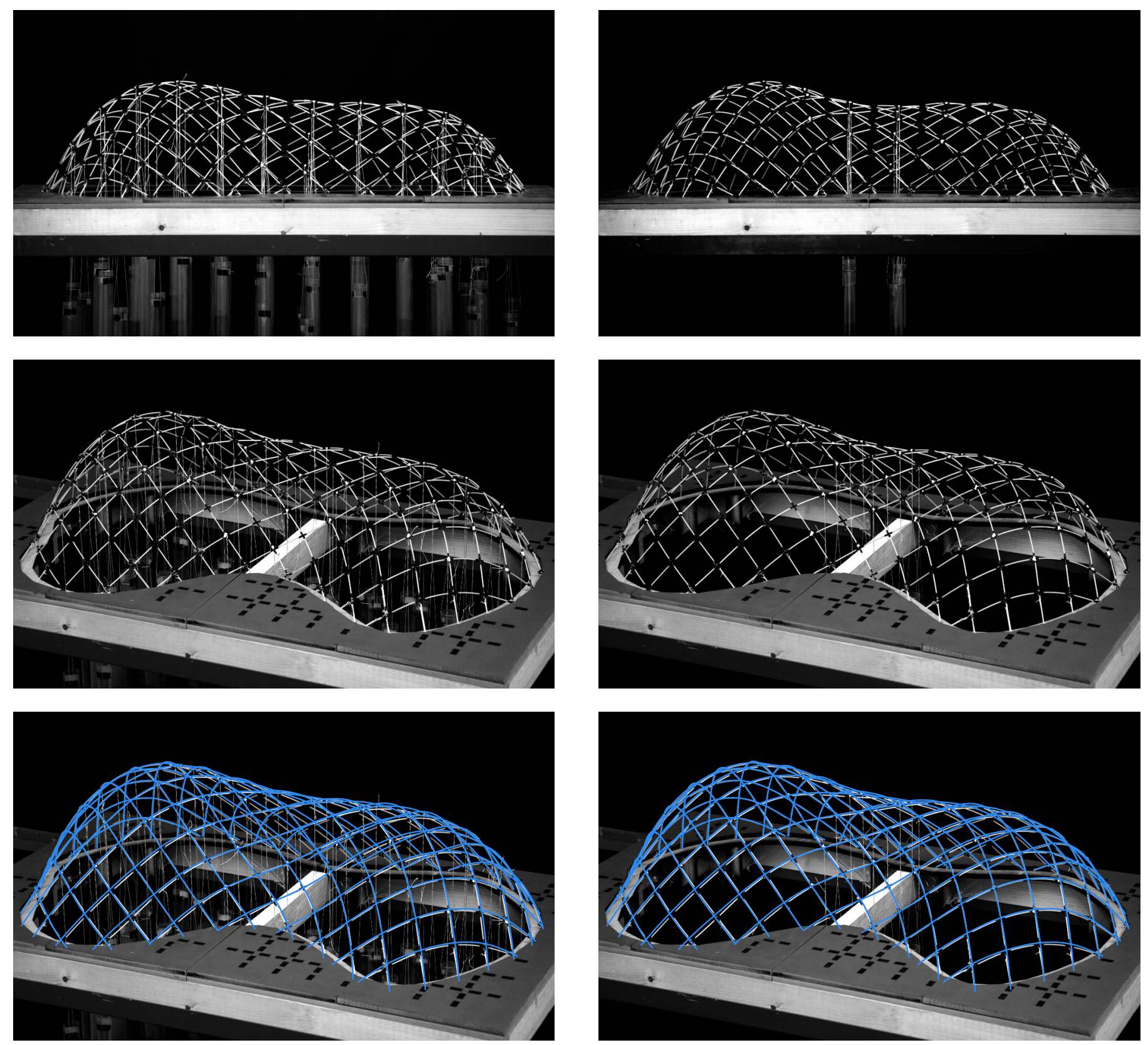

Figure 21: Geometry of the physical model without braces with $300 \mathrm{~g}$ on the nodes indicated in figure 9 , with the corresponding numerical model in blue.

Figure 22: Geometry of the physical model without braces with $500 \mathrm{~g}$ on the nodes indicated in figure 19, with the corresponding numerical model in blue. 


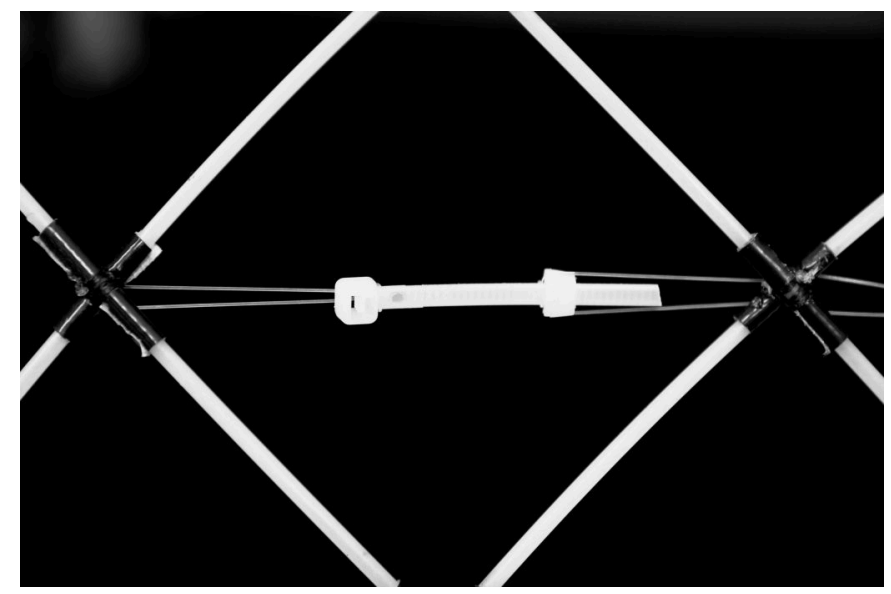

Figure 23: Braces are made from two double nylon wire strings and a tensioning system made from cable ties.

their initial lengths. A comparison of the numerical model and the physical model is shown in figure 24. Although the shape of the physical model matches the shape of the numerical model relatively well on first sight, the accuracy is slightly worse than before the cables were added, with deviations up to $14.0 \mathrm{~mm}$, with an average of $4.1 \mathrm{~mm}$.

Before loading the structure with the final design load, an additional test was performed first, applying the same load case as before, consisting of point loads of $300 \mathrm{~g}$. The response of the scale model shows a very similar mismatch as before the loads were applied, indicating that the relative displacements are quite accurately predicted by the numerical model (figure 25). Deviations range up to $16.3 \mathrm{~mm}$, with an average of 4.3 $\mathrm{mm}$ (table 2). As expected, adding bracing cables resulted in a much stiffer gridshell. Finally, the design load (point loads of $700 \mathrm{~g}$ ) was applied to the scale model. The difference between the numerical model and the physical model is a lot higher for this load case, which is clearly visible in figure 26. At this stage, the model was near collapse, and no additional scans were performed. It appears that the proposed optimization approach pushes the design to its limits, and, as a result, imperfections have a significant impact on the performance of the structure.

These imperfections were very hard to control for a model of such small scale. Several uncertainties influence the design, and it is hard to pinpoint the exact cause of the observed mismatch. Imperfections with respect to the consistency of the cable stiffness, irregularities in the grid layout, uncertainties in the pre-tension in the cables, small imperfections of the loads, dynamic effects, imperfections in support location and stiffness, variations in stiffness of the rods, creep effects, and the small inherent error in accuracy of the used beam model are all possible causes. It is expected that most of these imperfections can be drastically reduced if a gridshell is constructed on a larger scale, making use of a more informed technique to tension the bracing cables. Therefore, the next step should be to build a prototype on a larger scale, and find the most important sources of uncertainty. The impact of the remaining uncertainties can be reduced by means of robust design optimization [38]; one
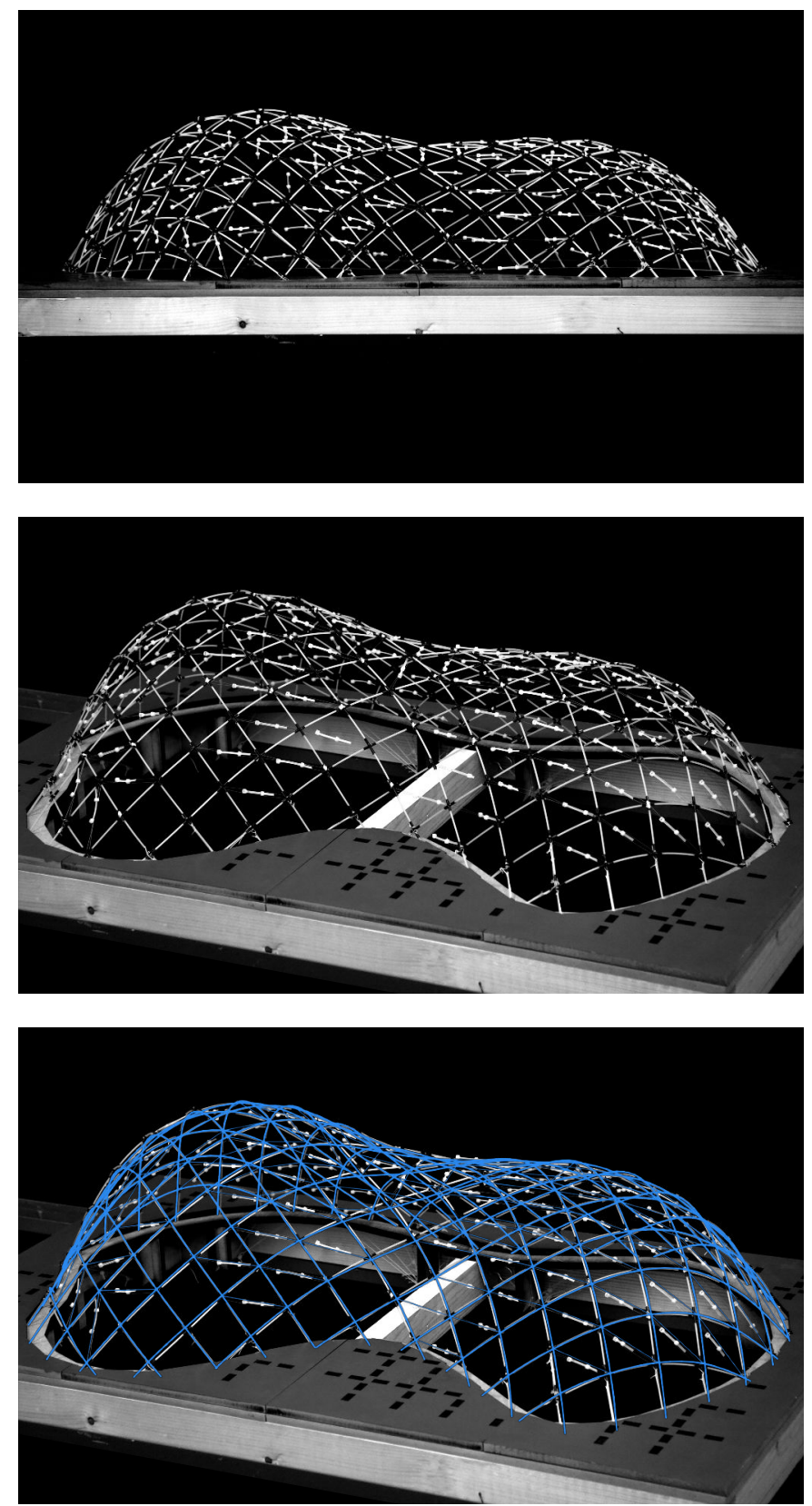

Figure 24: Geometry of the physical model with braces without loads, with the corresponding numerical model in blue. 

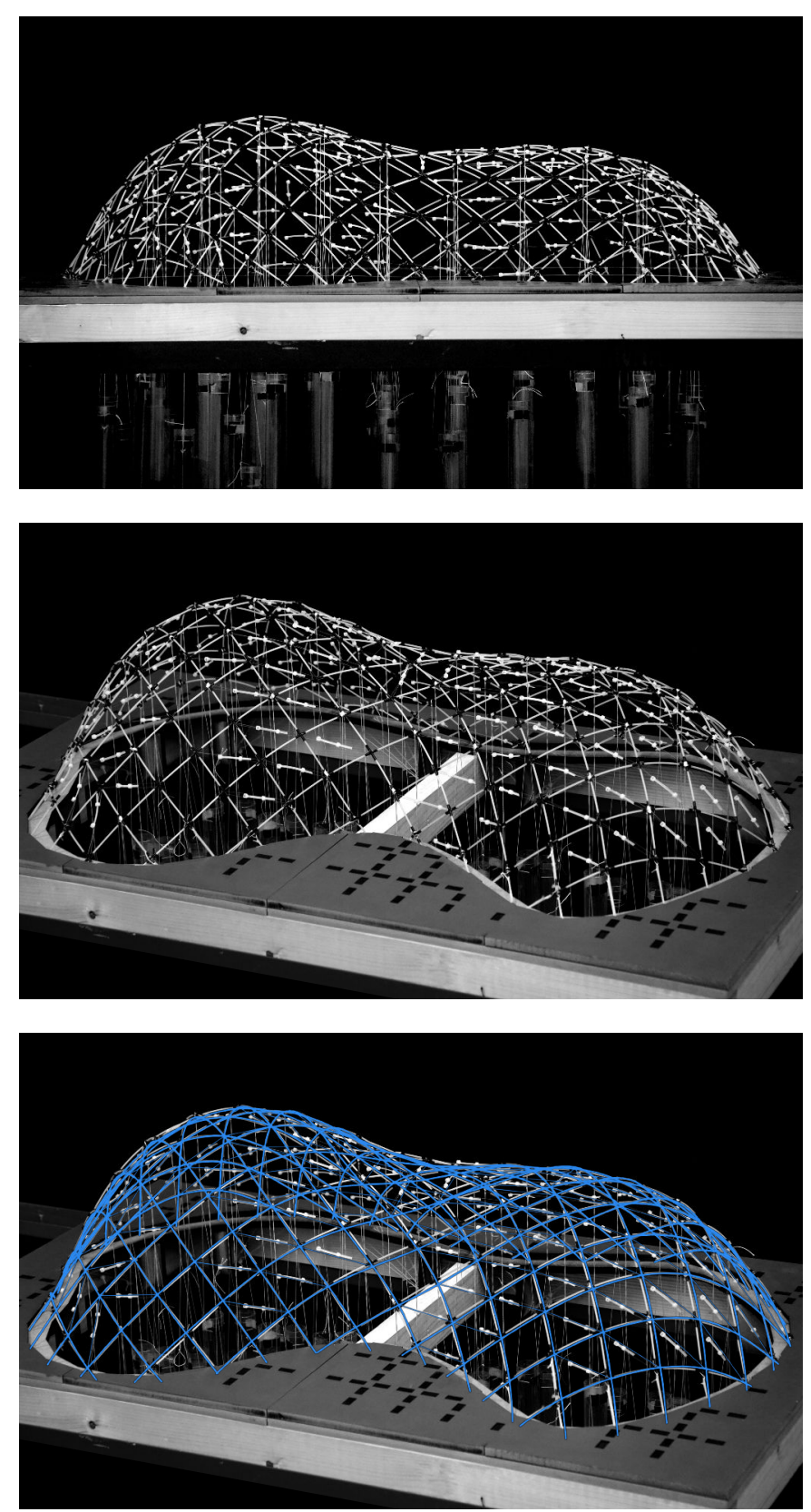

Figure 25: Geometry of the physical model with braces and with $300 \mathrm{~g}$ on the nodes indicated in figure 9 , with the corresponding numerical model in blue.
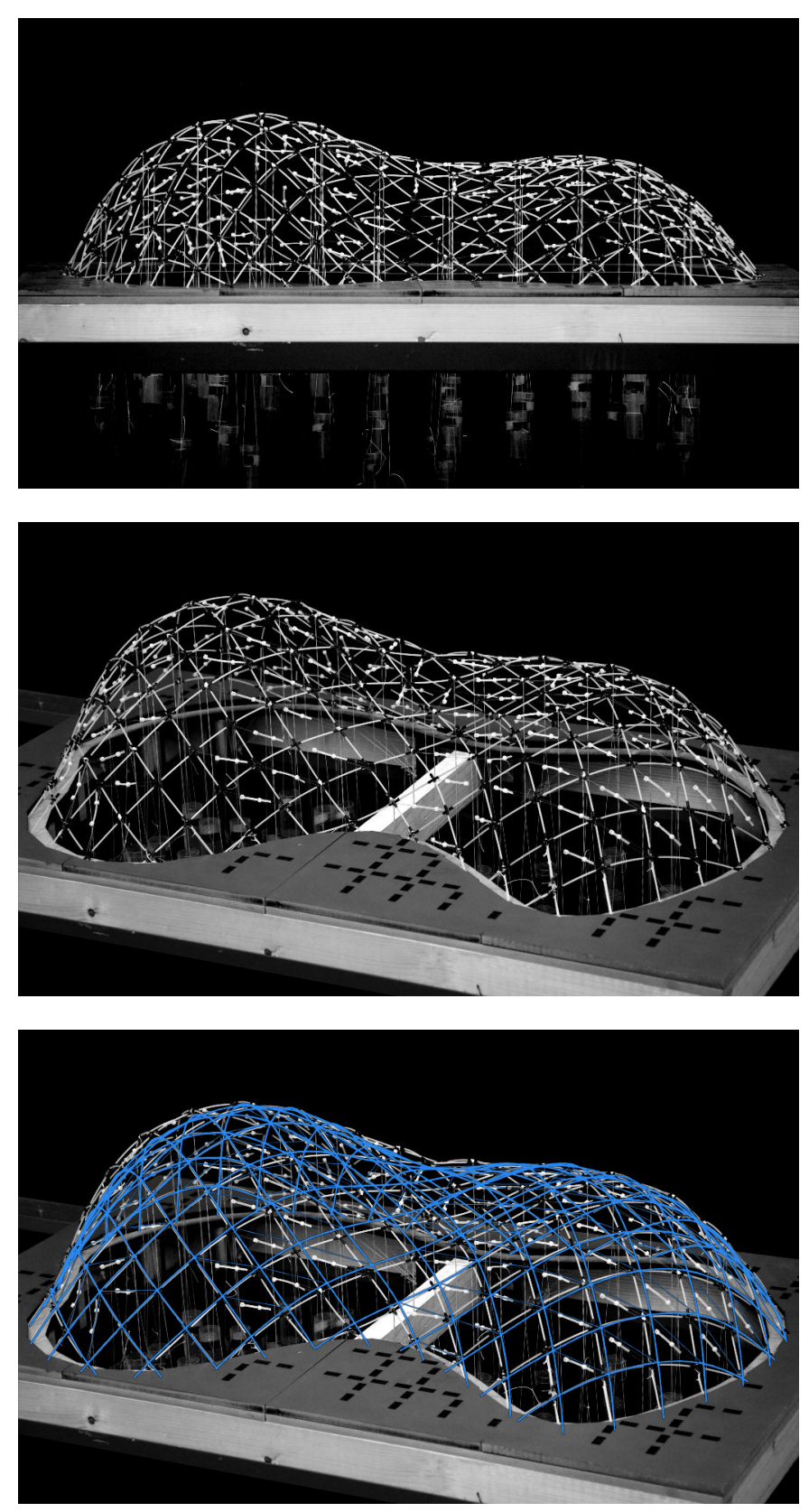

Figure 26: Geometry of the physical model with braces and with $700 \mathrm{~g}$ on the nodes indicated in figure 9, with the corresponding numerical model in blue.

$$
\text { (15) }
$$

Figure 27: Top view. 37 points are measured on the physical model to compare to the corresponding points of the numerical model. 
could e.g. define a joint probability distribution for all uncertain parameters and minimize the variance of the misfit between the shape of the loaded gridshell and the target shape.

Despite the observed deviations between the measured geometry and the expected geometry, some general conclusions and recommendations are made. Comparisons with smaller loads show a much better match, indicating that the method is more effective for stiffer designs, for example using stiffer cables, larger cross-sections or a denser grid. Additionally, it is worth investigating the effectiveness of taking more than one additional load case into account, simulating more asymmetric loading for example, to increase the robustness of the design. This would come at the cost of increased computation time, as a nonlinear structural analysis is required for every iteration of the optimization process for every considered load case. However, in practice, a sufficiently high accuracy and robustness are crucial, and are often worth the additional computational cost. Alternatively, the effectiveness of more thorough approaches to obtain a robust design, developed for other optimization problems could be explored [39]. However, some level of inaccuracy will always remain. Therefore, in addition to improving the accuracy and robustness of the design, the proposed optimization approach could be employed to post-tension the cables in the gridshell after construction, similar to the approach proposed by Liew et al. [10] for cable net falsework. This strategy requires an accurate real-time measuring system to control the grid geometry. Further research should clarify which adaptations are needed to the existing optimization setup to apply it in such an active control approach.

\section{Conclusions}

This paper presents a framework for the design of gridshells as falsework for concrete shells. As a key part of the design framework, a novel optimization approach was proposed to fit a gridshell to a given target shape, while taking external loading into account to make sure the gridshell fits the target shape when the concrete is applied. Practical constraints and penalization terms make sure the final design is feasible, and material use and construction time are minimized. A bracing system with adjustable lengths was suggested to allow control of the gridshell's shape after its relaxation. A scale model was constructed to test the design framework in practice and formulate recommendations for further research.

Numerical results showed promising improvements over existing design techniques on four areas; (1) deviations from the target shape were reduced, (2) the required number of braces was reduced, (3) the forces in the cables were effectively constrained, and (4) the robustness was improved by constraining deformations under additional loading. Furthermore, the crosssection of the grid rods can also be minimized. However, for practical reasons, this was not applied in the design of the scale model. In order for the optimization to be effective in practice, it is crucial that the numerical model accurately simulates the behavior of the physical model. Although the construction of a scale model showed a relatively good match for smaller loads, larger deviations were observed for the design load. Therefore, future research should focus on finding and eliminating the origin of the experienced inaccuracy, for example, by constructing a gridshell on a larger scale and accurately monitoring the gridshell's geometry and forces in the cables through all construction stages. Additionally, the potential of an active control system to adaptively control the cable lengths could be explored.

\section{Aknowledgements}

We would like to thank K. Langlois and T. Bacek for their assistance with scanning the scale model. This work was supported by the Research Foundation - Flanders (FWO; grant number G0C2315N).

\section{References}

[1] E. Ramm, K.-U. Bletzinger, R. Reitinger, Shape optimization of shell structures, Bulletin of the International Association for Shell and Spatial Structures 34 (2) (1993) 103-122. doi : 10.1080/12506559.1993. 10511083.

[2] A. Tomás, P. Martí, Shape and size optimisation of concrete shells, Engineering Structures 32 (6) (2010) 1650-1658. doi:10.1016/j. engstruct. 2010.02.013.

[3] K. Billig, Concrete shell roofs with flexible moulds, Journal of the ICE 25 (3) (1946) 228-231.

[4] B. Kromoser, P. Huber, Pneumatic formwork systems in structural engineering, Advances in Materials Science and Engineering 2016 (2016). doi: $10.1155 / 2016 / 4724036$.

[5] W. Sobek, Concrete shells constructed on pneumatic formwork, in: K. Heki (Ed.), Proceedings 1ASS Symposium, Vol. 1, 1986, pp. 337-344.

[6] W. Sobek, Auf Pneumatisch Gestützten Schalungen Hergestellte Betonschalen, Ursula Sobek, 1987.

[7] P. C. van Hennik, R. Houtman, Textile Composites and Inflatable Structures II., Vol. 8 of Computational Methods in Applied Sciences, Springer, 2008, Ch. Pneumatic Formwork for Irregular Curved Thin Shells, pp. 99116. doi:10.1007/978-1-4020-6856-0_7.

[8] T. Van Mele, P. Block, A novel form finding method for fabric formwork for concrete shells, Journal of the International Association for Shell and Spatial Structures 52 (4) (2011) 217-224.

[9] D. Veenendaal, P. Block, Design process for a prototype concrete shells using a hybrid cable-net and fabric formwork, Engineering Structures 75 (2014) 39-50. doi:10.1016/j . engstruct.2014.05.036.

[10] A. Liew, Y. R. Stürz, S. Guillaume, T. Van Mele, R. S. Smith, P. Block, Active control of a rod-net formwork system prototype, Automation in Construction 96 (2018) 128-140. doi:10.1016/j.autcon.2018.09. 002.

[11] P. Block, A. Schlueter, D. Veenendaal, J. Bakker, M. Begle, I. Hischier, J. Hofer, P. Jayathissa, I. Maxwell, T. Mendez Echenagucia, Z. Nagy, D. Pigram, B. Svetozarevic, R. Torsing, J. Verbeek, A. Willmann, G. Lydon, Nest hilo: Investigating lightweight construction and adaptive energy systems, Journal of Building Engineering 12 (2017) 332-341. doi : $10.1016 / j \cdot j$ jobe.2017.06.013.

[12] D. Veenendaal, J. Bakker, P. Block, Structural design of the flexibly formed, mesh-reinforced concrete sandwich shell roof of NEST Hilo, Journal of the International Association for Shell and Spatial Structures 58 (1) (2017) 23-38. doi : 10.20898/j. iass . 2017.191.847.

[13] M. Popescu, L. Reiter, A. Liew, T. Van Mele, R. Flatt, P. Block, Building in concrete with a knitted stay-in-place formwork: Prototype of a concrete shell bridge, Structures 14 (2018) 322-332. doi:10.1016/j . istruc. 2018.03.001.

[14] G. Tang, R. Pedreschi, Deployable gridshells as formwork for concrete shells, in: Proceedings of the International Society Of Flexible Formwork (ISOFF) Symposium 2015, 2015.

[15] L. Coar, M. Cox, S. Adriaenssens, L. De Laet, The design and construction of fabric formed ice shells with bending active frames utilizing principle stress patterns, in: A. Bögle, M. Grohmann (Eds.), Proceedings of the IASS Annual Symposium 2017, Hamburg, 2017. 
Table 2: Distance between numerical model and physical model for five scenarios measured at the points shown in figure 27.

\begin{tabular}{|c|c|c|c|c|c|}
\hline \multirow[b]{2}{*}{ pos. \case } & \multicolumn{5}{|c|}{ distance $[\mathrm{mm}]$} \\
\hline & 1 (fig. 20) & 2 (fig. 21) & 3 (fig. 22) & 4 (fig. 24) & 5 (fig. 25) \\
\hline 1 & 2.1 & 2.3 & 4.0 & 2.1 & 2.2 \\
\hline 2 & 4.9 & 5.0 & 2.1 & 1.5 & 1.4 \\
\hline 3 & 2.0 & 1.4 & 2.8 & 4.1 & 4.4 \\
\hline 4 & 2.4 & 3.3 & 0.9 & 4.2 & 4.0 \\
\hline 5 & 5.1 & 5.3 & 4.0 & 4.4 & 4.3 \\
\hline 6 & 4.0 & 4.8 & 1.8 & 3.1 & 2.8 \\
\hline 7 & 0.9 & 3.3 & 2.3 & 6.1 & 7.1 \\
\hline 8 & 2.1 & 3.3 & 2.7 & 2.1 & 2.7 \\
\hline 9 & 2.4 & 4.6 & 3.5 & 5.8 & 6.5 \\
\hline 10 & 3.0 & 4.8 & 3.4 & 3.6 & 3.6 \\
\hline 11 & 1.4 & 2.9 & 2.2 & 3.6 & 4.3 \\
\hline 12 & 2.5 & 3.6 & 3.7 & 5.6 & 5.1 \\
\hline 13 & 3.6 & 5.7 & 2.4 & 5.8 & 7.3 \\
\hline 14 & 3.0 & 3.3 & 3.4 & 2.0 & 2.3 \\
\hline 15 & 2.3 & 7.1 & 4.0 & 6.2 & 7.5 \\
\hline 16 & 7.3 & 8.8 & 4.0 & 3.4 & 4.9 \\
\hline 17 & 2.3 & 2.4 & 3.7 & 4.0 & 5.2 \\
\hline 18 & 3.8 & 1.8 & 4.4 & 3.3 & 3.0 \\
\hline 19 & 5.4 & 6.3 & 2.2 & 4.4 & 4.4 \\
\hline 20 & 5.6 & 6.2 & 4.7 & 3.9 & 3.0 \\
\hline 21 & 3.5 & 5.3 & 1.1 & 14.0 & 16.3 \\
\hline 22 & 2.3 & 6.1 & 8.0 & 12.5 & 9.7 \\
\hline 23 & 7.4 & 5.8 & 6.3 & 5.2 & 5.0 \\
\hline 24 & 1.7 & 2.8 & 2.4 & 2.5 & 1.5 \\
\hline 25 & 3.8 & 3.4 & 2.4 & 5.2 & 4.7 \\
\hline 26 & 3.3 & 5.8 & 5.1 & 4.3 & 3.4 \\
\hline 27 & 0.8 & 2.8 & 3.9 & 1.7 & 2.6 \\
\hline 28 & 2.2 & 3.8 & 4.6 & 1.8 & 1.9 \\
\hline 29 & 2.8 & 2.6 & 2.9 & 2.1 & 2.6 \\
\hline 30 & 2.4 & 1.8 & 1.5 & 1.5 & 1.9 \\
\hline 31 & 1.2 & 2.3 & 3.0 & 2.1 & 1.1 \\
\hline 32 & 3.8 & 4.3 & 1.8 & 2.0 & 2.8 \\
\hline 33 & 2.8 & 3.3 & 2.4 & 3.0 & 3.2 \\
\hline 34 & 3.1 & 3.8 & 2.5 & 3.7 & 4.1 \\
\hline 35 & 2.2 & 2.6 & 2.9 & 2.4 & 2.3 \\
\hline 36 & 2.8 & 4.3 & 2.7 & 3.0 & 3.3 \\
\hline 37 & 4.4 & 6.1 & 1.1 & 5.5 & 7.4 \\
\hline
\end{tabular}


[16] P. Cuvilliers, C. Douthe, L. Du Peloux, R. Le Roy, Hybrid structural skin: prototype of elastic gridshell braced with a concrete envelop, in: A. Bögle, M. Grohmann (Eds.), Proceedings of the IASS Annual Symposium 2017, Hamburg, 2017.

[17] E. L. Hernández, Design and optimisation of elastic gridshells, Ph.D. thesis, Universität der Künste Berlin (2015).

[18] E. Pignatelli, G. Mirra, S. Pone, InFormer: designing forming actions in post-formed gridshells by means of MOGAs, in: A. Bögle, M. Grohmann (Eds.), Proceedings of the IASS Annual Symposium, Hamburg, 2017.

[19] J. Rombouts, G. Lombaert, L. De Laet, M. Schevenels, A novel shape optimization approach for strained gridshells: design and construction of a simply supported gridshell, Engineering Structures 192 (2019) 166180 .

[20] F. Otto, E. Schauer, J. Hennicke, T. Hasegawa, IL 10 Gitterschalen / Grid shells: Bericht über das japanisch-deutsche Forschungsprojekt STI, durchgeführt von Mai 1971 vis Mai 1973 am Institut fur Leichte Flachentragwerke, Institute for Lightweight Structures, Stuttgart, 1974

[21] L. Bouhaya, O. Baverel, J.-F. Caron, Optimization of gridshell bar orientation using a simplified genetic approach, Structural and Multidisciplinary Optimization 50 (5) (2014) 839-848. doi:10.1007/ s00158-014-1088-9.

[22] S. Pone, G. Mirra, E. Pignatelli, D. Lancia, S. Colabella, Specialised algorithms for different project stages in a postformed timber gridshell design, in: Proceedings of the International Conference on Structures and Architecture, 2016. doi : 10.1201/b20891-33.

[23] E. L. Hernández, O. Baverel, C. Gengnagel, On the design and construction of elastic gridshells with irregular meshes, International Journal of Space Structures 28 (3-4) (2013) 161-174. doi : 10.1260/0266-3511. $28.3-4.161$

[24] L. Bouhaya, O. Baverel, J.-F. Caron, Mapping two-way continuous elastic grid on an imposed surface: Application to grid shells, in: A. Domingo, C. Lázaro (Eds.), Proceedings of the International Association for Shell and Spatial Structures (IASS) Symposium 2009, Valencia, 2009, pp. 989997.

[25] M. Kuijvenhoven, P. C. J. Hoogenboom, Particle-spring method for form finding grid shell structures consisting of flexible members, Journal of the International Association for Shell and Spatial Structures 53 (1) (2012) 31-38.

[26] J.-M. Li, J. Knippers, Designing regular and irregular elastic gridshells by six DOF dynamic relaxation, in: Proceedings of Design Modeling Symposium, Berlin, 2013.

[27] J. Rombouts, G. Lombaert, L. De Laet, M. Schevenels, A fast and accurate dynamic relaxation approach for form-finding and analysis of bending-active structures, International Journal of Space Structures 34 (1-2) (2019) 40-53. doi : 10 . 1177/0956059919864279.

[28] J. Rombouts, G. Lombaert, L. De Laet, M. Schevenels, On the equivalence of dynamic relaxation and the Newton-Raphson method, International Journal for Numerical Methods in Engineering 113 (9) (2018) 1531-1539. doi:10.1002/nme.5707.

[29] M. A. Crisfield, A consistent co-rotational formulation for nonlinear, three-dimensional, beam-elements, Computer Methods in Applied Mechanics and Engineering 81 (1990) 131-150. doi:10.1016/ 0045-7825 (90) 90106-V.

[30] T. Belytschko, L. Schwer, M. J. Klein, Large displacement, transient analysis of space frames, International Journal for Numerical Methods in Engineering 11 (1) (1977) 65-84. doi : 10.1002/nme.1620110108.

[31] M. A. Crisfield, Non-linear finite element analysis of solids and structures, Vol. 2, Wiley, 1997.

[32] C. A. Felippa, B. Haugen, A unified formulation of small-strain corotational finite elements: I. theory, Computer Methods in Applied Mechanics and Engineering 194 (2005) 2285-2335. doi:10.1016/j.cma. 2004. 07.035.

[33] M. Rippmann, L. Lachauer, P. Block, RhinoVAULT - interactive vault design, International Journal of Space Structures 27 (4) (2012) 219-230. doi:10.1260/0266-3511.27.4.219.

[34] P. Block, J. Ochsendorf, Thrust network analysis: A new methodology for three-dimensional equilibrium, Journal of the International Association for Shell and Spatial Structures 48 (3) (2007) 167-173.

[35] Vicon, Intelligence in motion, www.vicon.com (Accessed March 2019).

[36] K. Svanberg, The method of moving asymptotes - a new method for structural optimization, International Journal for Numerical Methods in
Engineering 24 (2) (1987) 359-373. doi:10.1002/nme.1620240207.

[37] P. Merriaux, Y. Dupuis, R. Boutteau, P. Vasseur, X. Savatier, A study of vicon system positioning performance, Sensors 17 (7) (2017). doi: $10.3390 / \mathrm{s} 17071591$.

[38] H. Beyer, B. Sendoff, Robust optimization - A comprehensive survey 196 (2007) 3190-3218.

[39] G. I. Schuëller, H. A. Jensen, Computational methods in optimization considering uncertainties - an overview, Computer Methods in Applied Mechanics and Engineering 198 (1) (2008) 2-13. doi:10.1016/ j . cma . 2008.05 .004 .

\section{Appendix A. Sensitivities}

In order to allow for the use of a gradient-based optimization algorithm, a sensitivity analysis of the optimization problem defined in section 2 is performed. Below, the objective function (equation (2)) and the left-hand side of constraints (5) and (6) are differentiated consecutively with respect to the undeformed cable lengths $\mathbf{I}_{c}^{0}$ and the radius of the cross-section of the grid rods $r_{\mathrm{b}}$.

\section{Appendix A.1. Differentiation with respect to the undeformed cable lengths}

In this subsection, the derivatives of the objective function (equation (2)) and the left-hand side of constraints (5) and (6) with respect to the undeformed cable lengths $\mathbf{l}_{\mathrm{c}}^{0}$ are first expressed in terms of the following three total derivatives: (1) the derivative $\mathrm{dd} / \mathrm{dl}_{\mathrm{c}}^{0}$ of the distance vector under the design load with respect to the undeformed cable lengths, (2) the derivative $\mathrm{d} \mathbf{d}_{1} / \mathrm{dl}_{\mathrm{c}}^{0}$ of the distance vector under the additional load case with respect to the undeformed cable lengths and (3) the derivative $\mathrm{dn}_{\mathrm{c}} / \mathrm{dl}_{\mathrm{c}}^{0}$ of the normal forces in the cables with respect to the undeformed cable lengths.

The following expression shows how the objective function $f$ is a function of the undeformed cable lengths $\mathbf{l}_{c}^{0}$ :

$$
\hat{f}\left(\mathbf{l}_{\mathrm{c}}^{0}\right)=f\left(\mathbf{d}\left(\mathbf{x}\left(\mathbf{l}_{\mathrm{c}}^{0}\right)\right), \mathbf{n}_{\mathrm{c}}\left(\mathbf{l}_{\mathrm{c}}^{0}, \mathbf{x}\left(\mathbf{l}_{\mathrm{c}}^{0}\right)\right)\right)
$$

where $\mathbf{d}$ is the distance vector, whose elements are defined in equation (7), $\mathbf{x}$ is a vector collecting the positional information of the nodes (i.e. coordinates and rotation vector components corresponding respectively to each translational degree of freedom and each rotational degree of freedom of the finite element model), and $\mathbf{n}_{\mathrm{c}}$ is the vector collecting the normal forces in the cables. Consequently, differentiation of the objective function with respect to the undeformed cable lengths gives:

$$
\frac{\mathrm{d} f}{\mathrm{~d} \mathbf{l}_{\mathrm{c}}^{0}}=\frac{\partial f}{\partial \mathbf{d}} \frac{\mathrm{dd}}{\mathrm{dl}_{\mathrm{c}}^{0}}+\frac{\partial f}{\partial \mathbf{n}_{\mathrm{c}}} \frac{\mathrm{d} \mathbf{n}_{\mathrm{c}}}{\mathrm{dl}_{\mathrm{c}}^{0}}
$$

where:

$$
\frac{\partial f}{\partial \mathbf{d}}=2 w_{1} \mathbf{d}^{\mathrm{T}}
$$

and $\partial f / \mathbf{n}_{\mathrm{c}}$ is elaborated for each cable $i$ individually:

$$
\frac{\partial f}{\partial n_{\mathrm{c}, i}}=w_{2}
$$


Differentiation of the left-hand side of constraint (5) also involves the derivation of $\mathrm{d} \mathbf{n}_{\mathrm{c}} / \mathrm{dl}_{\mathrm{c}}^{0}$. Differentiation of the left-hand side of constraint (6) gives:

$$
\frac{\mathrm{d}\left(\mathbf{d}_{1}^{\mathrm{T}} \mathbf{d}_{1}\right)}{\mathrm{d}_{\mathrm{c}}^{0}}=2 \mathbf{d}_{1}^{\mathrm{T}} \frac{\mathrm{d} \mathbf{d}_{1}}{\mathrm{dl}_{\mathrm{c}}^{0}}
$$

\section{Differentiation of the distance vector}

The distance vector $\mathbf{d}$ depends on the undeformed cable lengths as follows:

$$
\hat{\mathbf{d}}\left(\mathbf{l}_{\mathrm{c}}^{0}\right)=\mathbf{d}\left(\mathbf{x}\left(\mathbf{l}_{\mathrm{c}}^{0}\right)\right)
$$

Consequently, the derivative of the distance vector $\mathbf{d}$ is given by:

$$
\frac{\mathrm{d} \mathbf{d}}{\mathrm{dl}_{\mathrm{c}}^{0}}=\frac{\partial \mathbf{d}}{\partial \mathbf{x}} \frac{\mathrm{d} \mathbf{x}}{\mathrm{dl}_{\mathrm{c}}^{0}}
$$

where $\partial \mathbf{d} / \partial \mathbf{x}$ is elaborated for each node individually. For node $i$, differentiation of equation (7) gives:

$$
\frac{\partial d_{i}}{\partial \mathbf{x}}=\frac{\partial d_{i}}{\partial x_{i}} \frac{\mathrm{d} x_{i}}{\mathrm{~d} \mathbf{x}}+\frac{\partial d_{i}}{\partial y_{i}} \frac{\mathrm{d} y_{i}}{\mathrm{~d} \mathbf{x}}+\frac{\partial d_{i}}{\partial z_{i}} \frac{\mathrm{d} z_{i}}{\mathrm{~d} \mathbf{x}}
$$

The derivative of the distance $d_{i}$ with respect to the $\mathrm{x}$-coordinate of node $i$ is given by:

$$
\frac{\partial d_{i}}{\partial x_{i}}=\frac{x_{i}-x_{i}^{\mathrm{t}}}{d_{i}}
$$

where $x_{i}^{\mathrm{t}}$ is the $\mathrm{x}$-coordinate of node $i$ in the target grid. In equation (A.8), $\mathrm{d} x_{i} / \mathrm{d} \mathbf{x}$ can be written as:

$$
\frac{\mathrm{d} x_{i}}{\mathrm{~d} \mathbf{x}}=\mathbf{L}_{x_{i}}
$$

where $\mathbf{L}_{x_{i}}$ is a selection matrix selecting the x-coordinate $x_{i}$ of node $i$ from the position vector $\mathbf{x}$ :

$$
x_{i}=\mathbf{L}_{x_{i}} \mathbf{x}
$$

Similar expressions can be derived for the derivatives in terms of $y$-, and z-coordinates in equation (A.8).

The derivative of the position vector $\mathbf{x}$ with respect to the undeformed cable lengths $\mathbf{l}_{\mathrm{c}}^{0}$ in equation (A.7) is found by differentiation of equilibrium equation (8):

$$
\frac{\mathrm{d} \mathbf{r}}{\mathrm{dl}_{\mathrm{c}}^{0}}=\frac{\mathrm{d} \mathbf{p}}{\mathrm{dl}_{\mathrm{c}}^{0}}-\frac{\mathrm{d} \mathbf{f}}{\mathrm{dl}_{\mathrm{c}}^{0}}=\mathbf{0}
$$

where $\mathrm{d} \mathbf{p} / \mathrm{dl}^{0}$ is zero, as the undeformed cable lengths $\mathbf{l}_{\mathrm{c}}^{0}$ do not influence the magnitude of the loads, nor the degrees of freedom on which the loads act. On the other hand, the dependency of the internal forces $\mathbf{f}$ on the undeformed cable lengths $\mathbf{l}_{\mathrm{c}}^{0}$ is given by:

$$
\hat{\mathbf{f}}\left(\mathbf{l}_{\mathrm{c}}^{0}\right)=\mathbf{f}\left(\mathbf{l}_{\mathrm{c}}^{0}, \mathbf{x}\left(\mathbf{l}_{\mathrm{c}}^{0}\right)\right)
$$

Differentiation of the internal forces $\mathbf{f}$ results in:

$$
\frac{\mathrm{d} \mathbf{f}}{\mathrm{dl}_{\mathrm{c}}^{0}}=\frac{\partial \mathbf{f}}{\partial \mathbf{l}_{\mathrm{c}}^{0}}+\frac{\partial \mathbf{f}}{\partial \mathbf{x}} \frac{\mathrm{d} \mathbf{x}}{\mathrm{dl}_{\mathrm{c}}^{0}}
$$

Next, combining equations (A.12) and (A.14) gives:

$$
\frac{\partial \mathbf{f}}{\partial \mathbf{x}} \frac{\mathrm{d} \mathbf{x}}{\mathrm{dl}_{\mathrm{c}}^{0}}=-\frac{\partial \mathbf{f}}{\partial \mathbf{l}_{\mathrm{c}}^{0}}
$$

where $\partial \mathbf{f} / \partial \mathbf{x}$ is the tangential stiffness matrix. Equation (A.15) is rewritten as:

$$
\mathbf{K} \frac{\mathrm{d} \mathbf{x}}{\mathrm{d}_{\mathrm{c}}^{0}}=-\frac{\partial \mathbf{f}}{\partial \mathbf{l}_{\mathrm{c}}^{0}}
$$

which can be solved for $\mathrm{d} \mathbf{x} / \mathrm{dl}_{\mathrm{c}}^{0}$ when $\partial \mathbf{f} / \partial \mathbf{l}_{\mathrm{c}}^{0}$ is known. Differentiation of the internal forces $\mathbf{f}$ with respect to the undeformed cable lengths $\mathbf{l}_{\mathrm{c}}^{0}$ gives:

$$
\frac{\partial \mathbf{f}}{\partial \mathbf{l}_{\mathrm{c}}^{0}}=\sum_{i=1}^{k_{\mathrm{c}}} \frac{\partial \mathbf{f}}{\partial l_{i}^{0}} \frac{\mathrm{d} l_{i}^{0}}{\mathrm{~d} \mathbf{l}_{\mathrm{c}}^{0}}
$$

where $k_{\mathrm{c}}$ is the number of cables and $\mathrm{d} l_{i}^{0} / \mathrm{dl}_{\mathrm{c}}^{0}$ is a selection matrix $\mathbf{S}_{i}^{\mathrm{c}}$ selecting element $i$ from all cable elements:

$$
l_{i}^{0}=\mathbf{S}_{i}^{\mathrm{c}} \mathbf{l}_{\mathrm{c}}^{0}
$$

The derivative $\partial \mathbf{f} / \partial l_{i}^{0}$ in equation (A.17) is further elaborated:

$$
\frac{\partial \mathbf{f}}{\partial l_{i}^{0}}=\frac{\partial \mathbf{f}}{\partial \mathbf{f}_{i}^{e}} \frac{\partial \mathbf{f}_{i}^{e}}{\partial l_{i}^{0}}
$$

where $\mathbf{f}_{i}^{e}$ collects the internal forces of element $i$ and:

$$
\frac{\partial \mathbf{f}}{\partial \mathbf{f}_{i}^{e}}=\frac{\partial \mathbf{f}}{\partial \mathbf{f}_{\mathrm{F}}} \frac{\partial \mathbf{f}_{\mathrm{F}}}{\partial \mathbf{f}_{i}^{e}}=\mathbf{L}^{\mathrm{F}}\left(\mathbf{L}_{i}^{\mathrm{F}}\right)^{\mathrm{T}}
$$

where $\mathbf{f}_{\mathrm{F}}$ is the full internal force vector, collecting the internal forces corresponding to every degree of freedom, including fixed degrees of freedom, and $\mathbf{L}^{\mathrm{F}}$ and $\mathbf{L}_{i}^{\mathrm{F}}$ are selection matrices: $\mathbf{L}^{\mathrm{F}}$ selects the unconstrained degrees of freedom from all degrees of freedom and $\mathbf{L}_{i}^{\mathrm{F}}$ selects the degrees of freedom of element $i$ from all degrees of freedom:

$$
\mathbf{f}=\mathbf{L}^{\mathrm{F}} \mathbf{f}_{\mathrm{F}}
$$

$$
\mathbf{f}_{i}^{e}=\mathbf{L}_{i}^{\mathrm{F}} \mathbf{f}_{\mathrm{F}}
$$

The derivative $\partial \mathbf{f}_{i}^{e} / \partial l_{i}^{0}$ in equation (A.19) is found by differentiation of equation (19):

$$
\frac{\partial \mathbf{f}_{i}^{e}}{\partial l_{i}^{0}}=\frac{\partial \mathbf{T}_{i}^{e}}{\partial l_{i}^{0}} \overline{\mathbf{f}}_{i}^{e}+\mathbf{T}_{i}^{e} \frac{\partial \overline{\mathbf{f}}_{i}^{e}}{\partial l_{i}^{0}}
$$

where $\partial \mathbf{T}_{i}^{e} / \partial l_{i}^{0}$ is zero. Differentiation of equation (15) gives:

$$
\frac{\partial \overline{\mathbf{f}}_{i}^{e}}{\partial l_{i}^{0}}=\frac{\partial \overline{\mathbf{K}}_{i}^{e}}{\partial l_{i}^{0}} \overline{\mathbf{u}}_{i}^{e}+\overline{\mathbf{K}}_{i}^{e} \frac{\partial \overline{\mathbf{u}}_{i}^{e}}{\partial l_{i}^{0}}
$$

where the derivatives of $\overline{\mathbf{K}}_{i}^{e}$ and $\overline{\mathbf{u}}_{i}^{e}$ are found by differentiation of equations (18), and (13) and (17):

$$
\frac{\partial \overline{\mathbf{K}}_{i}^{e}}{\partial l_{i}^{0}}=-\frac{\overline{\mathbf{K}}_{i}^{e}}{l_{i}^{0}}
$$




$$
\frac{\partial \overline{\mathbf{u}}_{i}^{e}}{\partial l_{i}^{0}}=\left[\begin{array}{lllllll}
-1 & 0 & 0 & 0 & 0 & 0 & 0
\end{array}\right]^{\mathrm{T}}
$$

Inserting equations (A.25) and (A.26) into equation (A.24) gives $\partial \overline{\mathbf{f}}_{i}^{e} / \partial l_{i}^{0}$; which can be used in equation (A.23) to find $\partial \mathbf{f}_{i}^{e} / \partial l_{i}^{0}$. Inserting this derivative into equation (A.19), together with $\partial \mathbf{f} / \partial \mathbf{f}_{i}^{e}$ from equation (A.20) gives $\partial \mathbf{f} / \partial l_{i}^{0}$, which is required to solve equation (A.16), taking equation (A.17) and (A.18) into account. Finally, $\mathrm{dd} / \mathrm{dl}_{\mathrm{c}}^{0}$ is calculated by inserting $\mathrm{d} \mathbf{x} / \mathrm{dl}_{\mathrm{c}}^{0}$ from equation (A.16) and $\partial \mathbf{d} / \partial \mathbf{x}$ from equations (A.8) to (A.10) into equation (A.7). Differentiation of distance vector $\mathbf{d}_{1}$ follows along the same lines.

\section{Differentiation of the normal forces in the cables}

$\mathrm{Next}, \mathrm{d} \mathbf{n}_{\mathrm{c}} / \mathrm{dl}_{\mathrm{c}}^{0}$ is derived, which is required to determine the second term in equation (A.2), and for the differentiation of the left-hand side of constraint (5). The differentiation is performed for individual cables $i$ and $j$. The normal force $n_{\mathrm{c}, i}$ in cable $i$ depends on the undeformed cable length $l_{j}^{0}$ of cable $j$ as follows:

$$
\hat{n}_{\mathrm{c}, i}\left(l_{j}^{0}\right)=n_{\mathrm{c}, i}\left(\overline{\mathbf{f}}_{i}^{e}\left(\overline{\mathbf{u}}_{i}^{e}\left(l_{j}^{0}, \mathbf{x}_{i}^{e}\left(\mathbf{x}\left(l_{j}^{0}\right)\right)\right), \overline{\mathbf{K}}_{i}^{e}\left(l_{j}^{0}\right)\right)\right)
$$

Differentiation of the normal force $n_{\mathrm{c}, i}$ in cable $i$ with respect to the undeformed cable length of cable $j$ gives:

$$
\frac{\mathrm{d} n_{\mathrm{c}, i}}{\mathrm{~d} l_{j}^{0}}=\frac{\partial n_{\mathrm{c}, i}}{\partial \overline{\mathbf{f}}_{i}^{e}} \frac{\mathrm{d} \overline{\mathbf{f}}_{i}^{e}}{\mathrm{~d} l_{j}^{0}}
$$

where, because $n_{\mathrm{c}, i}=N_{i}$ is the first element of the internal force vector $\overline{\mathbf{f}}_{i}^{e}$ :

$$
\frac{\partial n_{\mathrm{c}, i}}{\partial \overline{\mathbf{f}}_{i}^{e}}=\left[\begin{array}{lllllll}
1 & 0 & 0 & 0 & 0 & 0 & 0
\end{array}\right]
$$

Next $\overline{\mathrm{f}}_{i}^{e} / \mathrm{d} l_{j}^{0}$ is further elaborated according to equation (15):

$$
\frac{\mathrm{d} \overline{\mathbf{f}}_{i}^{e}}{\mathrm{~d} l_{j}^{0}}=\frac{\mathrm{d} \overline{\mathbf{K}}_{i}^{e}}{\mathrm{~d} l_{j}^{0}} \overline{\mathbf{u}}_{i}^{e}+\overline{\mathbf{K}}_{i}^{e} \frac{\mathrm{d} \overline{\mathbf{u}}_{i}^{e}}{\mathrm{~d} l_{j}^{0}}
$$

where, if $j=i$, the total derivative $\mathrm{d} \overline{\mathbf{K}}_{i}^{e} / \mathrm{d} l_{j}^{0}$ is equal to the partial derivative $\partial \overline{\mathbf{K}}_{i}^{e} / \partial l_{i}^{0}$ given in equation (A.25), and if $j \neq i$, $\mathrm{d} \overline{\mathbf{K}}_{i}^{e} / \mathrm{d} l_{j}^{0}$ is zero. Next, the derivative $\mathrm{d} \overline{\mathbf{u}}_{i}^{e} / \mathrm{d} l_{j}^{0}$ is further elaborated:

$$
\frac{\mathrm{d} \overline{\mathbf{u}}_{i}^{e}}{\mathrm{~d} l_{j}^{0}}=\frac{\partial \overline{\mathbf{u}}_{i}^{e}}{\partial l_{j}^{0}}+\frac{\partial \overline{\mathbf{u}}_{i}^{e}}{\partial \mathbf{x}_{i}^{e}} \frac{\mathrm{d} \mathbf{x}_{i}^{e}}{\mathrm{~d} l_{j}^{0}}
$$

where, if $j=i, \partial \overline{\mathbf{u}}_{i}^{e} / \partial l_{j}^{0}$ is given by equation (A.26), and if $j \neq i, \partial \overline{\mathbf{u}}_{i}^{e} / \partial l_{j}^{0}$ is zero. Furthermore, $\partial \overline{\mathbf{u}}_{i}^{e} / \partial \mathbf{x}_{i}^{e}$ is the transformation matrix $\mathbf{T}_{i}^{e}$ defined in equation (20), and $\mathrm{d} \mathbf{x}_{i}^{e} / \mathrm{dl}_{j}^{0}$ is further elaborated:

$$
\frac{\mathrm{d} \mathbf{x}_{i}^{e}}{\mathrm{~d} l_{j}^{0}}=\frac{\partial \mathbf{x}_{i}^{e}}{\partial \mathbf{x}_{\mathrm{F}}} \frac{\mathrm{d} \mathbf{x}_{\mathrm{F}}}{\mathrm{d} l_{j}^{0}}
$$

where $\mathbf{x}_{\mathrm{F}}$ is the full position vector collecting all positional information for all degrees of freedom, including fixed degrees of freedom, and:

$$
\frac{\partial \mathbf{x}_{i}^{e}}{\partial \mathbf{x}_{\mathrm{F}}}=\mathbf{L}_{i}^{\mathrm{F}}
$$

where $\mathbf{L}_{i}^{\mathrm{F}}$ is the selection matrix introduced in equation (A.22). The next derivative in equation (A.32) is elaborated further:

$$
\frac{\mathrm{d} \mathbf{x}_{\mathrm{F}}}{\mathrm{d} l_{j}^{0}}=\frac{\partial \mathbf{x}_{\mathrm{F}}}{\partial \mathbf{x}} \frac{\mathrm{d} \mathbf{x}}{\mathrm{d} l_{j}^{0}}
$$

where $\mathrm{d} \mathbf{x} / \mathrm{d} l_{j}^{0}$ is obtained from equation (A.16), and:

$$
\frac{\partial \mathbf{x}_{\mathrm{F}}}{\partial \mathbf{x}}=\left(\mathbf{L}^{\mathrm{F}}\right)^{\mathrm{T}}
$$

where $\mathbf{L}^{\mathrm{F}}$ the selection matrix introduced in equation (A.21).

With $\partial \mathbf{x}_{\mathrm{F}} / \partial \mathbf{x}$ and $\mathrm{d} \mathbf{x} / \mathrm{dl}_{\mathrm{c}}^{0}$ from equations (A.35) and (A.16) respectively, $\mathrm{d} \mathbf{x}_{\mathrm{F}} / \mathrm{d} l_{j}^{0}$ can be calculated using equation (A.34). This derivative is then used in equation (A.32), together with $\partial \mathbf{x}_{i}^{e} / \partial \mathbf{x}_{\mathrm{F}}$ from equation (A.33) to obtain $\mathrm{d} \mathbf{x}_{i}^{e} / \mathrm{d} l_{j}^{0}$. Next, $\mathrm{d} \overline{\mathbf{u}}_{i}^{e} / \mathrm{d} l_{i}^{0}$ is calculated from equation (A.31) with $\partial \overline{\mathbf{u}}^{e} / \partial l_{j}^{0}$ and $\partial \overline{\mathbf{u}}_{i}^{e} / \partial \mathbf{x}_{i}^{e}$ from equations (A.26) and (20), respectively. The derivative $\mathrm{d} \overline{\mathbf{u}}_{i}^{e} / \mathrm{d} l_{j}^{0}$ is then used in equation (A.30) to find $\mathrm{d} \overline{\mathbf{f}}_{i}^{e} / \mathrm{d} l_{j}^{0}$ with $\mathrm{d} \overline{\mathbf{K}}_{i}^{e} / \mathrm{d} l_{j}^{0}$ from equation (A.25). Finally, $\mathrm{d} n_{\mathrm{c}, i} / \mathrm{d} l_{j}^{0}$ is calculated using equation (A.28) with the previously calculated $\mathrm{d} \overline{\mathbf{f}}_{i}^{e} / \mathrm{d} l_{j}^{0}$ and $\partial n_{\mathrm{c}, i} / \partial \overline{\mathbf{f}}_{i}^{e}$ from equation (A.29).

\section{Appendix A.2. Differentiation with respect to the radius of the cross-section of the grid rods}

Similar to the previous subsection, the derivatives of the objective function (equation (2)) and the left-hand side of constraints (5) and (6) with respect to the radius of the cross-section of the grid rods $r_{\mathrm{g}}$ are first expressed in terms of the following three total derivatives: (1) $\mathrm{d} \mathbf{d} / \mathrm{d} r_{\mathrm{g}}$, (2) $\mathrm{d} \mathbf{d}_{1} / \mathrm{d} r_{\mathrm{g}}$ and (3) $\mathrm{d} \mathbf{n}_{\mathrm{c}} / \mathrm{d} r_{\mathrm{g}}$. The calculation of these total derivatives themselves is tackled next.

The objective function $f$ is a function of the radius $r_{\mathrm{g}}$ of the cross-section of the grid rods as follows:

$$
\hat{f}\left(r_{\mathrm{g}}\right)=f\left(\mathbf{d}\left(\mathbf{x}\left(r_{\mathrm{g}}\right)\right), \mathbf{n}_{\mathrm{c}}\left(\mathbf{x}\left(r_{\mathrm{g}}\right)\right), r_{\mathrm{g}}\right)
$$

Differentiation of the objective function $f$ with respect to the radius $r_{\mathrm{g}}$ of the cross-section of the grid rods gives:

$$
\frac{\mathrm{d} f}{\mathrm{~d} r_{\mathrm{g}}}=\frac{\partial f}{\partial \mathbf{d}} \frac{\mathrm{d} \mathbf{d}}{\mathrm{d} r_{\mathrm{g}}}+\frac{\partial f}{\partial \mathbf{n}_{\mathrm{c}}} \frac{\mathrm{d} \mathbf{n}_{\mathrm{c}}}{\mathrm{d} r_{\mathrm{g}}}+\frac{\partial f}{\partial r_{\mathrm{g}}}
$$

where $\partial f / \partial \mathbf{d}$ and $\partial f / \partial \mathbf{n}_{\mathrm{c}}$ are defined in equations (A.3) and (A.4) respectively, and:

$$
\frac{\partial f}{\partial r_{\mathrm{g}}}=w_{3}
$$

Differentiation of the left-hand side of constraint (5) also involves the derivation of $\mathrm{d} \mathbf{n}_{\mathrm{c}} / \mathrm{d} r_{\mathrm{g}}$. Differentiation of the left hand side of constraint (6) gives:

$$
\frac{\mathrm{d}\left(\mathbf{d}_{1}^{\mathrm{T}} \mathbf{d}_{1}\right)}{\mathrm{d} r_{\mathrm{g}}}=\mathbf{d}_{1}^{\mathrm{T}} \frac{\mathrm{d} \mathbf{d}_{1}}{\mathrm{~d} r_{\mathrm{g}}}
$$

